\title{
On a coupled system of fractional sum-difference equations with $p$-Laplacian operator
}

\author{
Pimchana Siricharuanun ${ }^{1}$, Saowaluck Chasreechai ${ }^{2}$ and Thanin Sitthiwirattham ${ }^{3^{*}}$ (I)
}

\section{"Correspondence:}

thanin_sit@dusit.ac.th

${ }^{3}$ Mathematics Department, Faculty

of Science and Technology, Suan

Dusit University, Bangkok 10300,

Thailand

Full list of author information is

available at the end of the article

\begin{abstract}
In this paper, we propose a nonlocal fractional sum-difference boundary value problem for a coupled system of fractional sum-difference equations with $p$-Laplacian operator. The problem contains both Riemann-Liouville and Caputo fractional difference with five fractional differences and four fractional sums. The existence and uniqueness result of the problem is studied by using the Banach fixed point theorem.
\end{abstract}

MSC: 39A05; 39A12

Keywords: $p$-Laplacian operator; Existence and uniqueness; Coupled system of fractional difference equations; Boundary value problem

\section{Introduction}

Discrete fractional calculus and fractional difference equations have been widely studied. Goodrich and Peterson gave some useful basic definitions and properties of fractional difference calculus in the book [1]. Discrete fractional calculus can be applied in queuing problems, economics, logistic map, and electrical networks, see [2-4]. The extension of discrete fractional calculus has helped to build up some of the basic theory in this area, see [5-32] and the references cited therein.

The boundary value problem for fractional differential equations and the system of equations with $p$-Laplacian operator were presented in [33-39] and [40-44], respectively. Particularly, the boundary value problem for fractional difference equations with $p$-Laplacian operator was presented in [45-47]. In addition, the existence results of systems of fractional boundary value problems were presented in [48-55].

We observe that the boundary value problem of a coupled system of nonlinear fractional difference equations with $p$-Laplacian operator has not been studied. This result is the motivation for this research. In this paper, we aim to study the coupled system of nonlinear fractional sum-difference equations with $p$-Laplacian operator

$$
\begin{aligned}
\Delta_{C}^{\alpha_{1}} \phi_{p}\left[\Delta_{C}^{\beta_{1}} u_{1}(t)\right]= & F_{1}\left[t+\alpha_{1}+\beta_{1}-1, t+\alpha_{2}+\beta_{2}-1, \Delta^{\gamma_{1}} u_{1}\left(t+\alpha_{1}+\beta_{1}-\gamma_{1}\right),\right. \\
& \left.\Psi^{\omega_{2}} u_{2}\left(t+\alpha_{2}+\beta_{2}+\omega_{2}-1\right), u_{2}\left(t+\alpha_{2}+\beta_{2}+\omega_{2}-1\right)\right],
\end{aligned}
$$

(c) The Author(s) 2020. This article is licensed under a Creative Commons Attribution 4.0 International License, which permits use, sharing, adaptation, distribution and reproduction in any medium or format, as long as you give appropriate credit to the original author(s) and the source, provide a link to the Creative Commons licence, and indicate if changes were made. The images or other third party material in this article are included in the article's Creative Commons licence, unless indicated otherwise in a credit line to the material. If material is not included in the article's Creative Commons licence and your intended use is not permitted by statutory regulation or exceeds the permitted use, you will need to obtain permission directly from the copyright holder. To view a copy of this licence, visit http://creativecommons.org/licenses/by/4.0/. 


$$
\begin{aligned}
\Delta_{C}^{\alpha_{2}} \phi_{p}\left[\Delta_{C}^{\beta_{2}} u_{2}(t)\right]= & F_{2}\left[t+\alpha_{2}+\beta_{2}-1, t+\alpha_{1}+\beta_{1}-1, \Delta^{\gamma_{2}} u_{2}\left(t+\alpha_{2}+\beta_{2}-\gamma_{2}\right),\right. \\
& \left.\Psi^{\omega_{1}} u_{1}\left(t+\alpha_{1}+\beta_{1}+\omega_{1}-1\right), u_{1}\left(t+\alpha_{1}+\beta_{1}+\omega_{1}-1\right)\right]
\end{aligned}
$$

with the nonlocal fractional sum and fractional difference boundary conditions

$$
\begin{array}{ll}
\Delta_{C}^{\beta_{1}} u_{1}\left(\alpha_{1}-1\right)=0, & u_{1}\left(T+\alpha_{1}+\beta_{1}\right)=\lambda_{2} \Delta^{-\theta_{2}} g_{2}\left(\eta_{2}+\theta_{2}\right) u_{2}\left(\eta_{2}+\theta_{2}\right), \\
\Delta_{C}^{\beta_{2}} u_{2}\left(\alpha_{2}-1\right)=0, & u_{2}\left(T+\alpha_{2}+\beta_{2}\right)=\lambda_{1} \Delta^{-\theta_{1}} g_{1}\left(\eta_{1}+\theta_{1}\right) u_{1}\left(\eta_{1}+\theta_{1}\right),
\end{array}
$$

where $t \in \mathbb{N}_{0, T}:=\{0,1, \ldots, T\}, \alpha_{i}, \beta_{i}, \gamma_{i}, \omega_{i}, \theta_{i} \in(0,1), \alpha_{i}+\beta_{i} \in(1,2], \quad \lambda_{i}>0, \eta_{i} \in$ $\mathbb{N}_{\alpha_{i}+\beta_{i}-1, T+\alpha_{i}+\beta_{i}-1}, F_{i} \in C\left(\mathbb{N}_{\alpha_{1}+\beta_{1}-2, T+\alpha_{1}+\beta_{1}} \times \mathbb{N}_{\alpha_{2}+\beta_{2}-2, T+\alpha_{2}+\beta_{2}} \times \mathbb{R}^{3}, \mathbb{R}\right), g_{i} \in C\left(\mathbb{N}_{\alpha_{i}+\beta_{i}-2, T+\alpha_{i}+\beta_{i}}\right.$, $\mathbb{R}^{+}$), and for $\varphi_{i}: \mathbb{N}_{\alpha_{1}+\beta_{1}-2, T+\alpha_{1}+\beta_{1}} \times \mathbb{N}_{\alpha_{2}+\beta_{2}-2, T+\alpha_{2}+\beta_{2}} \rightarrow[0, \infty)$,

$$
\begin{aligned}
\Psi^{\omega_{i}} u_{i}\left(t+\omega_{i}\right) & :=\left[\Delta^{-\omega_{i}} \varphi_{i} u_{i}\right]\left(t+\omega_{i}\right) \\
& =\frac{1}{\Gamma\left(\omega_{i}\right)} \sum_{s=\alpha_{i}+\beta_{i}-\omega_{i}-2}^{t-\omega_{i}}(t-\sigma(s)) \frac{\omega_{i}-1}{\varphi_{i}}\left(t, s+\omega_{i}\right) u_{i}\left(s+\omega_{i}\right)
\end{aligned}
$$

for $i \in\{1,2\}$. For $p>1$, the $p$-Laplacian operator is defined as $\phi_{p}(x)=|x|^{p-2} x$, where $\phi_{p}$ is invertible and its inverse operator is $\phi_{q}$, where $q>1$ is a constant such that $\frac{1}{p}+\frac{1}{q}=1$.

Our plan is as follows. In Sect. 2, we recall some basic knowledge and convert (1.1)-(1.2) to an equivalent summation equation and find its solution. In Sect. 3, we prove existence and uniqueness of the solution of boundary value problem (1.1)-(1.2) by using the Banach fixed point theorem. Some examples to illustrate our result are presented in the last section.

\section{Preliminaries}

Notations, definitions, and lemmas which are used in the main results are given as follows.

Definition 2.1 The generalized falling function is defined by $t^{\underline{\alpha}}:=\frac{\Gamma(t+1)}{\Gamma(t+1-\alpha)}$ for any $t$ and $\alpha$ for which the right-hand side is defined. If $t+1-\alpha$ is a pole of the gamma function and $t+1$ is not a pole, then $t^{\underline{\alpha}}=0$.

Lemma 2.1 ([5]) Assume that the following factorial functions are well defined:

(i) $(t-\mu) t_{\underline{\mu}}=t \stackrel{\mu+1}{\underline{n}}$, where $\mu \in \mathbb{R}$.

(ii) If $t \leq r$, then $t^{\underline{\alpha}} \leq r^{\underline{\alpha}}$ for any $\alpha>0$.

Definition 2.2 Let $\alpha>0$ and $f$ be defined on $\mathbb{N}_{a}$, the $\alpha$-order fractional sum of $f$ is defined by

$$
\Delta^{-\alpha} f(t):=\frac{1}{\Gamma(\alpha)} \sum_{s=a}^{t-\alpha}(t-\sigma(s))^{\frac{\alpha-1}{} f(s)}
$$

where $t \in \mathbb{N}_{a+\alpha}$ and $\sigma(s)=s+1$.

Definition 2.3 For $\alpha>0$ and $f$ defined on $\mathbb{N}_{a}$, the $\alpha$-order Riemann-Liouville fractional difference of $f$ is defined by

$$
\Delta^{\alpha} f(t):=\Delta^{N} \Delta^{-(N-\alpha)} f(t)=\frac{1}{\Gamma(-\alpha)} \sum_{s=a}^{t+\alpha}(t-\sigma(s))^{\frac{-\alpha-1}{}} f(s) .
$$


The $\alpha$-order Caputo fractional difference of $f$ is defined by

$$
\Delta_{C}^{\alpha} f(t):=\Delta^{-(N-\alpha)} \Delta^{N} f(t)=\frac{1}{\Gamma(N-\alpha)} \sum_{s=a}^{t-(N-\alpha)}(t-\sigma(s))^{\frac{N-\alpha-1}{2}} \Delta^{N} f(s),
$$

where $t \in \mathbb{N}_{a+N-\alpha}$ and $N \in \mathbb{N}$ is chosen so that $0 \leq N-1<\alpha<N$. If $\alpha=N$, then $\Delta^{\alpha} f(t)=$ $\Delta_{C}^{\alpha} f(t)=\Delta^{N} f(t)$.

Lemma $2.2([7])$ Let $0 \leq N-1<\alpha \leq N$. Then

$$
\Delta^{-\alpha} \Delta_{C}^{\alpha} y(t)=y(t)+C_{0}+C_{1} t+\cdots+C_{N-1} t \underline{N-1}
$$

for some $C_{i} \in \mathbb{R}$, with $1 \leq i \leq N$.

We provide some properties of the $p$-Laplacian operator as follows.

(A1) If $1<p<2, x y>0$ and $|x|,|y| \geq m>0$, then

$$
\left|\phi_{p}(x)-\phi_{p}(y)\right| \leq(p-1) m^{p-2}|x-y|
$$

(A2) If $p>2, x y>0$ and $|x|,|y| \leq M$, then

$$
\left|\phi_{p}(x)-\phi_{p}(y)\right| \leq(p-1) M^{p-2}|x-y| .
$$

Next, we find a solution of the linear variant of boundary value problem (1.1)-(1.2) as shown in the following lemma.

Lemma 2.3 For $i, j \in\{1,2\}$ and $i \neq j$, let $\Lambda \neq 0, \alpha_{i}, \beta_{i}, \theta_{i} \in(0,1), \alpha_{i}+\beta_{i} \in(1,2], \lambda_{i}>0$ be given constants, $h_{i} \in C\left(\mathbb{N}_{\alpha_{i}+\beta_{i}-2, T+\alpha_{i}+\beta_{i}}, \mathbb{R}\right)$ and $g_{i} \in C\left(\mathbb{N}_{\alpha_{i}+\beta_{i}-2, T+\alpha_{i}+\beta_{i}}, \mathbb{R}^{+}\right)$be given functions. Then the linear variant problem given by

$$
\begin{aligned}
& \Delta_{C}^{\alpha_{i}} \phi_{p}\left[\Delta_{C}^{\beta_{i}} u_{i}(t)\right]=h_{i}\left(t+\alpha_{i}-1\right), \quad t \in \mathbb{N}_{0, T}, \\
& \Delta_{C}^{\beta_{i}} u_{i}\left(\alpha_{i}-1\right)=0, \\
& u_{i}\left(T+\alpha_{i}+\beta_{i}\right)=\lambda_{j} \Delta^{-\theta_{j}} g_{j}\left(\eta_{j}+\theta_{j}\right) u_{j}\left(\eta_{j}+\theta_{j}\right), \quad \eta_{j} \in \mathbb{N}_{\alpha_{j}+\beta_{j}-1, T+\alpha_{j}+\beta_{j}-1}
\end{aligned}
$$

has the unique solution $\left(u_{1}, u_{2}\right)$, where

$$
\begin{aligned}
u_{1}\left(t_{1}\right)= & \frac{1}{\Gamma\left(\beta_{1}\right)} \sum_{s=\alpha_{1}-1}^{t_{1}-\beta_{1}}\left(t_{1}-\sigma(s)\right)^{\frac{\beta_{1}-1}{}} \phi_{q}\left[\frac{1}{\Gamma\left(\alpha_{1}\right)} \sum_{\xi=0}^{s-\alpha_{1}}(s-\sigma(\xi))^{\frac{\alpha_{1}-1}{}} h_{1}\left(\xi+\alpha_{1}+\beta_{1}-1\right)\right] \\
& +\frac{1}{\Lambda}\left\{\left(\frac{\lambda_{1}}{\Gamma\left(\theta_{1}\right)} \sum_{s=\alpha_{1}+\beta_{1}-2}^{\eta_{1}}\left(\eta_{1}+\theta_{1}-\sigma(s)\right)^{\frac{\theta_{1}-1}{}} g_{1}(s)\right) \mathcal{P}\left[h_{1}, h_{2}\right]+\mathcal{Q}\left[h_{1}, h_{2}\right]\right\}, \\
u_{2}\left(t_{2}\right)= & \frac{1}{\Gamma\left(\beta_{2}\right)} \sum_{s=\alpha_{2}-1}^{t_{2}-\beta_{2}}\left(t_{2}-\sigma(s)\right)^{\frac{\beta_{2}-1}{}} \phi_{q}\left[\frac{1}{\Gamma\left(\alpha_{2}\right)} \sum_{\xi=0}^{s-\alpha_{2}}(s-\sigma(\xi))^{\frac{\alpha_{2}-1}{h}} h_{2}\left(\xi+\alpha_{2}+\beta_{2}-1\right)\right] \\
& +\frac{1}{\Lambda}\left\{\left(\frac{\lambda_{2}}{\Gamma\left(\theta_{2}\right)} \sum_{s=\alpha_{2}+\beta_{2}-2}^{\eta_{2}}\left(\eta_{2}+\theta_{2}-\sigma(s)\right)^{\frac{\theta_{1}-1}{g_{2}}} g_{2}(s)\right) \mathcal{Q}\left[h_{1}, h_{2}\right]+\mathcal{P}\left[h_{1}, h_{2}\right]\right\},
\end{aligned}
$$


where $t_{i} \in \mathbb{N}_{\alpha_{i}+\beta_{i}-2, T+\alpha_{i}+\beta_{i}}$, the constant $\Lambda$ is defined by

$$
\begin{aligned}
\Lambda= & \frac{\lambda_{1} \lambda_{2}}{\Gamma\left(\theta_{1}\right) \Gamma\left(\theta_{2}\right)} \sum_{s=\alpha_{1}+\beta_{1}-1}^{\eta_{1}}\left(\eta_{1}+\theta_{1}-\sigma(s)\right) \frac{\theta_{1}-1}{g_{1}}(s) \\
& \times \sum_{s=\alpha_{2}+\beta_{2}-1}^{\eta_{2}}\left(\eta_{2}+\theta_{2}-\sigma(s)\right)^{\frac{\theta_{2}-1}{}} g_{2}(s)-1,
\end{aligned}
$$

and the functionals $\mathcal{P}\left[h_{1}, h_{2}\right], \mathcal{Q}\left[h_{1}, h_{2}\right]$ are defined by

$$
\begin{aligned}
& \mathcal{P}\left[h_{1}, h_{2}\right] \\
& =\frac{1}{\Gamma\left(\beta_{1}\right)} \sum_{s=\alpha_{1}-1}^{T+\alpha_{1}}\left(T+\alpha_{1}+\beta_{1}-\sigma(s)\right) \frac{\beta_{1}-1}{} \\
& \times \phi_{q}\left[\frac{1}{\Gamma\left(\alpha_{1}\right)} \sum_{\xi=0}^{s-\alpha_{1}}(s-\sigma(\xi))^{\frac{\alpha_{1}-1}{2}} h_{1}\left(\xi+\alpha_{1}+\beta_{1}-1\right)\right] \\
& -\frac{\lambda_{2}}{\Gamma\left(\beta_{2}\right) \Gamma\left(\theta_{2}\right)} \sum_{r=\alpha_{2}+\beta_{2}-1}^{\eta_{2}} \sum_{s=\alpha_{2}-1}^{r-\beta_{2}}\left(\eta_{2}+\theta_{2}-\sigma(r)\right) \frac{\theta_{2}-1}{}(r-\sigma(s))^{\frac{\beta_{2}-1}{}} g_{2}(r) \\
& \times \phi_{q}\left[\frac{1}{\Gamma\left(\alpha_{2}\right)} \sum_{\xi=0}^{s-\alpha_{2}}(s-\sigma(\xi)) \frac{\alpha_{2}-1}{h_{2}}\left(\xi+\alpha_{2}+\beta_{2}-1\right)\right], \\
& =\frac{1}{\Gamma\left(\beta_{2}\right)} \sum_{s=\alpha_{2}-1}^{T+\alpha_{2}}\left(T+\alpha_{2}+\beta_{2}-\sigma(s)\right)^{\underline{\beta_{2}-1}} \\
& \times \phi_{q}\left[\frac{1}{\Gamma\left(\alpha_{2}\right)} \sum_{\xi=0}^{s-\alpha_{2}}(s-\sigma(\xi)) \frac{\alpha_{2}-1}{h_{2}} h_{2}\left(\xi+\alpha_{2}+\beta_{2}-1\right)\right] \\
& -\frac{\lambda_{1}}{\Gamma\left(\beta_{1}\right) \Gamma\left(\theta_{1}\right)} \sum_{r=\alpha_{1}+\beta_{1}-1}^{\eta_{1}} \sum_{s=\alpha_{1}-1}^{r-\beta_{1}}\left(\eta_{1}+\theta_{1}-\sigma(r)\right) \frac{\theta_{1}-1}{}(r-\sigma(s))^{\frac{\beta_{1}-1}{}} g_{1}(r) \\
& \times \phi_{q}\left[\frac{1}{\Gamma\left(\alpha_{1}\right)} \sum_{\xi=0}^{s-\alpha_{1}}(s-\sigma(\xi))^{\frac{\alpha_{1}-1}{}} h_{1}\left(\xi+\alpha_{1}+\beta_{1}-1\right)\right] \text {. }
\end{aligned}
$$

Proof For $i, j \in\{1,2\}$ and $i \neq j$, taking the fractional sum of order $\alpha_{i}$ for (2.1), we have

$$
\phi_{p}\left[\Delta_{C}^{\beta_{i}} u_{i}(t)\right]=C_{0 i}+\frac{1}{\Gamma\left(\alpha_{i}\right)} \sum_{s=0}^{t-\alpha_{i}}(t-\sigma(s))^{\frac{\alpha_{i}-1}{}} h_{i}\left(s+\alpha_{i}+\beta_{i}-1\right)
$$

for $t \in \mathbb{N}_{\alpha_{i}-1, T+\alpha_{i}}$.

From boundary condition (2.2), it implies that

$$
C_{0 i}=0 .
$$


Then from (2.9) we have

$$
\Delta_{C}^{\beta_{i}} u_{i}(t)=\phi_{q}\left[\frac{1}{\Gamma\left(\alpha_{i}\right)} \sum_{s=0}^{t-\alpha_{i}}(t-\sigma(s)) \frac{\alpha_{i}-1}{h_{i}}\left(s+\alpha_{i}+\beta_{i}-1\right)\right]
$$

Next, taking the fractional sum of order $\beta_{i}$ for (2.10), we have

$$
\begin{aligned}
u_{i}(t)= & C_{1 i}+\frac{1}{\Gamma\left(\beta_{i}\right)} \sum_{s=\alpha_{i}-1}^{t-\beta_{i}}(t-\sigma(s))^{\frac{\beta_{i}-1}{}} \\
& \times \phi_{q}\left[\frac{1}{\Gamma\left(\alpha_{i}\right)} \sum_{\xi=0}^{s-\alpha_{i}}(s-\sigma(\xi))^{\frac{\alpha_{i}-1}{}} h_{i}\left(\xi+\alpha_{i}+\beta_{i}-1\right)\right]
\end{aligned}
$$

for $t \in \mathbb{N}_{\alpha_{i}+\beta_{i}-2, T+\alpha_{i}+\beta_{i}}$.

Using the fractional sum of order $\theta_{i}$ for (2.11), we get

$$
\begin{aligned}
\Delta^{-\theta_{i}} u(t)= & \frac{C_{1 i}}{\Gamma\left(\theta_{i}\right)} \sum_{s=\alpha_{i}+\beta_{i}-2}^{t-\theta_{i}}(t-\sigma(s)) \frac{\theta_{i}-1}{} \\
& +\frac{1}{\Gamma\left(\theta_{i}\right) \Gamma\left(\beta_{i}\right)} \sum_{r=\alpha_{i}+\beta_{i}-1}^{t-\theta_{i}} \sum_{s=\alpha_{i}-1}^{t-\beta_{i}}(t-\sigma(r)) \frac{\theta_{i}-1}{(r-\sigma(s)) \frac{\beta_{i}-1}{}} \\
& \times \phi_{q}\left[\frac{1}{\Gamma\left(\alpha_{i}\right)} \sum_{\xi=0}^{s-\alpha_{i}}(s-\sigma(\xi)) \frac{\alpha_{i}-1}{h_{i}} h_{i}\left(\xi+\alpha_{i}+\beta_{i}-1\right)\right]
\end{aligned}
$$

for $t \in \mathbb{N}_{\alpha_{i}+\beta_{i}+\theta_{i}-3, T+\alpha_{i}+\beta_{i}+\theta_{i}}$.

Using boundary condition (2.3) implies

$$
\begin{aligned}
C_{11}- & C_{12} \frac{\lambda_{2}}{\Gamma\left(\theta_{2}\right)} \sum_{s=\alpha_{2}+\beta_{2}-2}^{\eta_{2}}\left(\eta_{2}+\theta_{2}-\sigma(s)\right)^{\frac{\theta_{2}-1}{}} g_{2}(s) \\
= & \frac{\lambda_{2}}{\Gamma\left(\theta_{2}\right) \Gamma\left(\beta_{2}\right)} \sum_{r=\alpha_{2}+\beta_{2}-1}^{\eta_{2}} \sum_{s=\alpha_{2}-1}^{r-\beta_{2}}\left(\eta_{2}+\theta_{2}-\sigma(r)\right)^{\frac{\theta_{2}-1}{}}(r-\sigma(s))^{\frac{\beta_{2}-1}{}} g_{2}(r) \\
& \times \phi_{q}\left[\frac{1}{\Gamma\left(\alpha_{2}\right)} \sum_{\xi=0}^{s-\alpha_{2}}(s-\sigma(\xi))^{\frac{\alpha_{2}-1}{}} h_{2}\left(\xi+\alpha_{2}+\beta_{2}-1\right)\right] \\
& -\frac{1}{\Gamma\left(\beta_{1}\right)} \sum_{s=\alpha_{1}-1}^{T+\alpha_{1}}\left(T+\alpha_{1}+\beta_{1}-\sigma(s)\right)^{\frac{\beta_{1}-1}{s-\alpha_{1}}} \\
& \times \phi_{q}\left[\frac{1}{\Gamma\left(\alpha_{1}\right)} \sum_{\xi=0}(s-\sigma(\xi))^{\frac{\alpha_{1}-1}{n}} h_{1}\left(\xi+\alpha_{1}+\beta_{1}-1\right)\right]
\end{aligned}
$$


and

$$
\begin{aligned}
C_{21}- & C_{22} \frac{\lambda_{1}}{\Gamma\left(\theta_{1}\right)} \sum_{s=\alpha_{1}+\beta_{1}-2}^{\eta_{1}}\left(\eta_{1}+\theta_{1}-\sigma(s)\right)^{\frac{\theta_{1}-1}{}} g_{1}(s) \\
= & \frac{\lambda_{1}}{\Gamma\left(\theta_{1}\right) \Gamma\left(\beta_{1}\right)} \sum_{r=\alpha_{1}+\beta_{1}-1}^{\eta_{1}} \sum_{s=\alpha_{1}-1}^{r-\beta_{1}}\left(\eta_{1}+\theta_{1}-\sigma(r)\right)^{\frac{\theta_{1}-1}{}}(r-\sigma(s))^{\frac{\beta_{1}-1}{}} g_{1}(r) \\
& \times \phi_{q}\left[\frac{1}{\Gamma\left(\alpha_{1}\right)} \sum_{\xi=0}^{s-\alpha_{1}}(s-\sigma(\xi))^{\frac{\alpha_{1}-1}{}} h_{1}\left(\xi+\alpha_{1}+\beta_{1}-1\right)\right] \\
& \left.-\frac{1}{\Gamma\left(\beta_{2}\right)} \sum_{s=\alpha_{2}-1}^{T+\alpha_{2}}\left(T+\alpha_{2}+\beta_{2}-\sigma(s)\right)^{\frac{\beta_{2}-1}{s-\alpha_{2}}}\right] \\
& \times \phi_{q}\left[\frac{1}{\Gamma\left(\alpha_{2}\right)} \sum_{\xi=0}(s-\sigma(\xi))^{\frac{\alpha_{2}-1}{h}} h_{2}\left(\xi+\alpha_{2}+\beta_{2}-1\right)\right] .
\end{aligned}
$$

$C_{11}, C_{12}$ can be represented by solving equations (2.13) and (2.14) as

$$
C_{11}=\frac{1}{\Lambda}\left\{\left(\frac{\lambda_{1}}{\Gamma\left(\theta_{1}\right)} \sum_{s=\alpha_{1}+\beta_{1}-2}^{\eta_{1}}\left(\eta_{1}+\theta_{1}-\sigma(s)\right)^{\frac{\theta_{1}-1}{}} g_{1}(s)\right) \mathcal{P}\left[h_{1}, h_{2}\right]+\mathcal{Q}\left[h_{1}, h_{2}\right]\right\}
$$

and

$$
C_{12}=\frac{1}{\Lambda}\left\{\left(\frac{\lambda_{2}}{\Gamma\left(\theta_{2}\right)} \sum_{s=\alpha_{2}+\beta_{2}-2}^{\eta_{2}}\left(\eta_{2}+\theta_{2}-\sigma(s)\right)^{\frac{\theta_{1}-1}{}} g_{2}(s)\right) \mathcal{Q}\left[h_{1}, h_{2}\right]+\mathcal{P}\left[h_{1}, h_{2}\right]\right\},
$$

where $\Lambda, \mathcal{P}\left(h_{1}, h_{2}\right)$ and $\mathcal{Q}\left(h_{1}, h_{2}\right)$ are defined as (2.6)-(2.8), respectively.

After substituting $C_{11}$ and $C_{12}$ into (2.11), we obtain (2.4) and (2.5).

\section{Existence and uniqueness result}

In this section, we study the existence and uniqueness result for problem (1.1)-(1.2). For each $i, j \in\{1,2\}$ and $i \neq j$, we let $E_{i}: C\left(\mathbb{N}_{\alpha_{i}+\beta_{i}-2, T+\alpha_{i}+\beta_{i}}, \mathbb{R}\right)$ be the Banach space for all functions on $\mathbb{N}_{\alpha_{i}+\beta_{i}-2, T+\alpha_{i}+\beta_{i}}$. Clearly, the product space $\mathcal{C}=E_{1} \times E_{2}$ is the Banach space. Define the spaces

$$
\mathcal{C}_{i}=\left\{\left(u_{1}, u_{2}\right) \in \mathcal{C}: \Delta^{\gamma_{i}} u_{i}\left(t_{i}-\gamma_{i}+1\right) \in E_{i}\right\}, \quad t_{i} \in \mathbb{N}_{\alpha_{i}+\beta_{i}-2, T+\alpha_{i}+\beta_{i}},
$$

with the norm

$$
\left\|\left(u_{1}, u_{2}\right)\right\|_{\mathcal{C}_{i}}=\max \left\{\left\|\Delta^{\gamma_{i}} u_{i}\right\|,\left\|u_{j}\right\|\right\}
$$

where

$$
\left\|\Delta^{\gamma_{i}} u_{i}\right\|=\max _{t_{i} \in \mathbb{N}_{\alpha_{i}+\beta_{i}-2, T+\alpha_{i}+\beta_{i}}}\left|\Delta^{\gamma_{i}} u_{i}\left(t_{i}-\gamma_{i}+1\right)\right| \quad \text { and } \quad\left\|u_{j}\right\|=\max _{t_{j} \in \mathbb{N}_{\alpha_{j}+\beta_{j}-2, T+\alpha_{j}+\beta_{j}}}\left|u_{j}\left(t_{j}\right)\right| .
$$

Obviously, the space $\left(\mathcal{C}_{1} \cap \mathcal{C}_{2},\left\|\left(u_{1}, u_{2}\right)\right\|_{\mathcal{C}_{1} \cap \mathcal{C}_{2}}\right)$ is also the Banach space with the norm

$$
\left\|\left(u_{1}, u_{2}\right)\right\|_{\mathcal{C}_{1} \cap \mathcal{C}_{2}}=\max \left\{\left\|\left(u_{1}, u_{2}\right)\right\|_{\mathcal{C}_{1}},\left\|\left(u_{1}, u_{2}\right)\right\|_{\mathcal{C}_{2}}\right\}
$$


Let $\mathcal{U}=\mathcal{C}_{1} \cap \mathcal{C}_{2}$. The operator $\mathcal{T}: \mathcal{U} \rightarrow \mathcal{U}$ is defined by

$$
\left(\mathcal{T}\left(u_{1}, u_{2}\right)\right)\left(t_{1}, t_{2}\right)=\left(\left(\mathcal{T}_{1}\left(u_{1}, u_{2}\right)\right)\left(t_{1}, t_{2}\right),\left(\mathcal{T}_{2}\left(u_{1}, u_{2}\right)\right)\left(t_{1}, t_{2}\right)\right)
$$

and

$$
\begin{aligned}
& \left(\mathcal{T}_{1}\left(u_{1}, u_{2}\right)\right)\left(t_{1}, t_{2}\right) \\
& =\frac{1}{\Gamma\left(\beta_{1}\right)} \sum_{s=\alpha_{1}-1}^{t_{1}-\beta_{1}}\left(t_{1}-\sigma(s)\right) \underline{\underline{\beta_{1}-1}} \\
& \times \phi_{q}\left[\frac{1}{\Gamma\left(\alpha_{1}\right)} \sum_{\xi=\alpha_{1}+\beta_{1}-1}^{s+\beta_{1}-1}\left(s+\alpha_{1}+\beta_{1}-1-\sigma(\xi)\right) \frac{\alpha_{1}-1}{} F_{1}^{*}\left[u\left(t_{2}, \xi\right)\right]\right]
\end{aligned}
$$

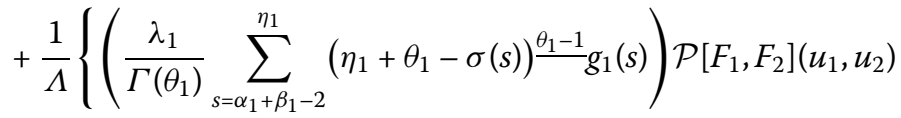

$$
\begin{aligned}
& \left.+\mathcal{Q}\left[F_{1}, F_{2}\right]\left(u_{1}, u_{2}\right)\right\} \\
& \left(\mathcal{T}_{2}\left(u_{1}, u_{2}\right)\right)\left(t_{1}, t_{2}\right) \\
& =\frac{1}{\Gamma\left(\beta_{2}\right)} \sum_{s=\alpha_{2}-1}^{t_{2}-\beta_{2}}\left(t_{2}-\sigma(s)\right) \frac{\beta_{2}-1}{} \\
& \times \phi_{q}\left[\frac{1}{\Gamma\left(\alpha_{2}\right)} \sum_{\xi=\alpha_{2}+\beta_{2}-1}^{s+\beta_{2}-1}\left(s+\alpha_{2}+\beta_{2}-1-\sigma(\xi)\right)^{\frac{\alpha_{2}-1}{2}} F_{2}^{*}\left[u\left(t_{1}, \xi\right)\right]\right]
\end{aligned}
$$

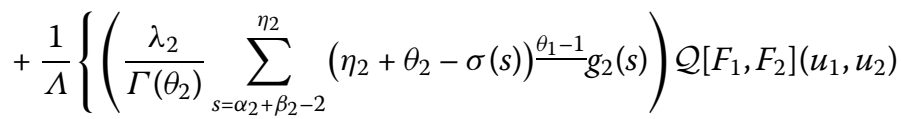

$$
\begin{aligned}
& \left.+\mathcal{P}\left[F_{1}, F_{2}\right]\left(u_{1}, u_{2}\right)\right\}
\end{aligned}
$$

where $t_{i} \in \mathbb{N}_{\alpha_{i}+\beta_{i}-2, T+\alpha_{i}+\beta_{i}}, \Lambda$ is defined as (2.6), and the functionals $\mathcal{P}\left[F_{1}, F_{2}\right]\left(u_{1}, u_{2}\right)$, $\mathcal{Q}\left[F_{1}, F_{2}\right]\left(u_{1}, u_{2}\right)$ are defined by

$$
\begin{aligned}
\mathcal{P}\left[F_{1}, F_{2}\right]\left(u_{1}, u_{2}\right) & \frac{1}{\Gamma\left(\beta_{1}\right)} \sum_{s=\alpha_{1}-1}^{T+\alpha_{1}}\left(T+\alpha_{1}+\beta_{1}-\sigma(s)\right)^{\frac{\beta_{1}-1}{}} \\
& \times \phi_{q}\left[\frac{1}{\Gamma\left(\alpha_{1}\right)} \sum_{\xi=\alpha_{1}+\beta_{1}-1}^{s+\beta_{1}-1}\left(s+\alpha_{1}+\beta_{1}-1-\sigma(\xi)\right)^{\frac{\alpha_{1}-1}{2}} F_{1}^{*}\left[u\left(t_{2}, \xi\right)\right]\right] \\
& -\frac{\lambda_{2}}{\Gamma\left(\beta_{2}\right) \Gamma\left(\theta_{2}\right)} \sum_{r=\alpha_{2}+\beta_{2}-1}^{\eta_{2}} \sum_{s=\alpha_{2}-1}^{r-\beta_{2}}\left(\eta_{2}+\theta_{2}-\sigma(r)\right)^{\frac{\theta_{2}-1}{}}(r-\sigma(s))^{\frac{\beta_{2}-1}{2}} g_{2}(r) \\
& \times \phi_{q}\left[\frac{1}{\Gamma\left(\alpha_{2}\right)} \sum_{\xi=\alpha_{2}+\beta_{2}-1}\left(s+\alpha_{2}+\beta_{2}-1-\sigma(\xi)\right)^{\frac{\alpha_{2}-1}{}} F_{2}^{*}\left[u\left(t_{1}, \xi\right)\right]\right]
\end{aligned}
$$




$$
\begin{aligned}
\mathcal{Q}\left[F_{1}, F_{2}\right]\left(u_{1}, u_{2}\right) & \\
= & \frac{1}{\Gamma\left(\beta_{2}\right)} \sum_{s=\alpha_{2}-1}^{T+\alpha_{2}}\left(T+\alpha_{2}+\beta_{2}-\sigma(s)\right)^{\frac{\beta_{2}-1}{}} \\
& \times \phi_{q}\left[\frac{1}{\Gamma\left(\alpha_{2}\right)} \sum_{\xi=\alpha_{2}+\beta_{2}-1}^{s+\beta_{2}-1}\left(s+\alpha_{2}+\beta_{2}-1-\sigma(\xi)\right)^{\frac{\alpha_{2}-1}{}} F_{2}^{*}\left[u\left(t_{1}, \xi\right)\right]\right] \\
& -\frac{\lambda_{1}}{\Gamma\left(\beta_{1}\right) \Gamma\left(\theta_{1}\right)} \sum_{r=\alpha_{1}+\beta_{1}-1}^{\eta_{1}} \sum_{s=\alpha_{1}-1}^{r-\beta_{1}}\left(\eta_{1}+\theta_{1}-\sigma(r)\right)^{\frac{\theta_{1}-1}{}}(r-\sigma(s))^{\frac{\beta_{1}-1}{}} g_{1}(r) \\
& \times \phi_{q}\left[\frac{1}{\Gamma\left(\alpha_{1}\right)} \sum_{\xi=\alpha_{1}+\beta_{1}-1}^{s+\beta_{1}-1}\left(s+\alpha_{1}+\beta_{1}-1-\sigma(\xi)\right)^{\frac{\alpha_{1}-1}{}} F_{1}^{*}\left[u\left(t_{2}, \xi\right)\right],\right.
\end{aligned}
$$

with

$$
F_{i}^{*}\left[u\left(t_{j}, \xi\right)\right]=F_{i}\left[t_{j}, \xi, \Delta^{\gamma_{i}} u_{i}\left(\xi-\gamma_{i}+1\right), \Psi^{\omega_{j}} u_{j}\left(t_{j}+\omega_{j}\right), u_{j}\left(t_{j}\right)\right] .
$$

For each $i, j \in\{1,2\}$ and $i \neq j$, we define the operators $\left(\mathcal{T}_{i}^{0}\left(u_{1}, u_{2}\right)\right)\left(t_{1}, t_{2}\right)$ and $\left(\mathcal{T}_{i}^{*}\left(u_{1}, u_{2}\right)\right)\left(t_{1}\right.$, $\left.t_{2}\right)$ by

$$
\begin{aligned}
& \left(\mathcal{T}_{1}^{0}\left(u_{1}, u_{2}\right)\right)\left(t_{1}, t_{2}\right)=\phi_{q}\left[\sum_{\xi=\alpha_{1}+\beta_{1}-1}^{t_{1}+\beta_{1}-1} \frac{\left(t_{1}+\alpha_{1}+\beta_{1}-1-\sigma(\xi)\right)^{\alpha_{1}-1}}{\Gamma\left(\alpha_{1}\right)} F_{1}^{*}\left[u\left(t_{2}, \xi\right)\right]\right] \\
& \left(\mathcal{T}_{2}^{0}\left(u_{1}, u_{2}\right)\right)\left(t_{1}, t_{2}\right)=\phi_{q}\left[\sum_{\xi=\alpha_{2}+\beta_{2}-1}^{t_{2}+\beta_{2}-1} \frac{\left(t_{2}+\alpha_{2}+\beta_{2}-1-\sigma(\xi)\right)^{\alpha_{2}-1}}{\Gamma\left(\alpha_{2}\right)} F_{2}^{*}\left[u\left(t_{1}, \xi\right)\right],\right.
\end{aligned}
$$

and

$$
\begin{aligned}
\left(\mathcal{T}_{1}^{*}\left(u_{1}, u_{2}\right)\right)\left(t_{1}, t_{2}\right) & \\
= & \frac{1}{\Gamma\left(\beta_{1}\right)} \sum_{s=\alpha_{1}-1}^{t_{1}-\beta_{1}}\left(t_{1}-\sigma(s)\right)^{\frac{\beta_{1}-1}{}}\left(u_{1}, u_{2}\right)\left(t_{2}, s\right)+\frac{1}{\Lambda}\left\{\mathcal{Q}^{*}\left[F_{1}, F_{2}\right]\left(u_{1}, u_{2}\right)\right. \\
& \left.+\left(\frac{\lambda_{1}}{\Gamma\left(\theta_{1}\right)} \sum_{s=\alpha_{1}+\beta_{1}-2}^{\eta_{1}}\left(\eta_{1}+\theta_{1}-\sigma(s)\right)^{\frac{\theta_{1}-1}{}} g_{1}(s)\right) \mathcal{P}^{*}\left[F_{1}, F_{2}\right]\left(u_{1}, u_{2}\right)\right\}, \\
\left(\mathcal{T}_{2}^{*}\left(u_{1}, u_{2}\right)\right)\left(t_{1}, t_{2}\right) & \\
= & \frac{1}{\Gamma\left(\beta_{2}\right)} \sum_{s=\alpha_{2}-1}^{t_{2}-\beta_{2}}\left(t_{2}-\sigma(s)\right)^{\frac{\beta_{2}-1}{}}\left(u_{1}, u_{2}\right)\left(t_{1}, s\right)+\frac{1}{\Lambda}\left\{\mathcal{P}^{*}\left[F_{1}, F_{2}\right]\left(u_{1}, u_{2}\right)\right. \\
& \left.+\left(\frac{\lambda_{2}}{\Gamma\left(\theta_{2}\right)} \sum_{s=\alpha_{2}+\beta_{2}-2}^{\eta_{2}}\left(\eta_{2}+\theta_{2}-\sigma(s)\right)^{\frac{\theta_{1}-1}{g_{2}}} g_{2}(s)\right) \mathcal{Q}^{*}\left[F_{1}, F_{2}\right]\left(u_{1}, u_{2}\right)\right\},
\end{aligned}
$$


where $t_{i} \in \mathbb{N}_{\alpha_{i}+\beta_{i}-2, T+\alpha_{i}+\beta_{i}}$, and the functionals $\mathcal{P}^{*}\left[F_{1}, F_{2}\right]\left(u_{1}, u_{2}\right), \mathcal{Q}^{*}\left[F_{1}, F_{2}\right]\left(u_{1}, u_{2}\right)$ are defined by

$$
\begin{aligned}
& \mathcal{P}^{*}\left[F_{1}, F_{2}\right]\left(u_{1}, u_{2}\right) \\
& =\frac{1}{\Gamma\left(\beta_{1}\right)} \sum_{s=\alpha_{1}-1}^{T+\alpha_{1}}\left(T+\alpha_{1}+\beta_{1}-\sigma(s)\right)^{\frac{\beta_{1}-1}{2}}\left(u_{1}, u_{2}\right)\left(t_{2}, s\right) \\
& \quad-\frac{\lambda_{2}}{\Gamma\left(\beta_{2}\right) \Gamma\left(\theta_{2}\right)} \sum_{r=\alpha_{2}+\beta_{2}-1}^{\eta_{2}} \sum_{s=\alpha_{2}-1}^{r-\beta_{2}}\left(\eta_{2}+\theta_{2}-\sigma(r)\right)^{\frac{\theta_{2}-1}{}}(r-\sigma(s))^{\frac{\beta_{2}-1}{}} \\
& \quad \times g_{2}(r)\left(u_{1}, u_{2}\right)\left(t_{1}, s\right), \\
& \mathcal{Q}^{*}\left[F_{1}, F_{2}\right]\left(u_{1}, u_{2}\right) \\
& =\frac{1}{\Gamma\left(\beta_{2}\right)} \sum_{s=\alpha_{2}-1}^{T+\alpha_{2}}\left(T+\alpha_{2}+\beta_{2}-\sigma(s)\right)^{\frac{\beta_{2}-1}{2}}\left(u_{1}, u_{2}\right)\left(t_{1}, s\right) \\
& \quad-\frac{\lambda_{1}}{\Gamma\left(\beta_{1}\right) \Gamma\left(\theta_{1}\right)} \sum_{r=\alpha_{1}+\beta_{1}-1}^{\eta_{1}} \sum_{s=\alpha_{1}-1}^{r-\beta_{1}}\left(\eta_{1}+\theta_{1}-\sigma(r)\right)^{\frac{\theta_{1}-1}{(}}(r-\sigma(s))^{\frac{\beta_{1}-1}{}} \\
& \quad \times g_{1}(r)\left(u_{1}, u_{2}\right)\left(t_{2}, s\right) .
\end{aligned}
$$

Let $\mathcal{T}_{i}=\mathcal{T}_{i}{ }^{*} \circ \mathcal{T}_{i}^{0}$, then $\mathcal{T}_{i}$ and $\mathcal{T}: \mathcal{U} \rightarrow \mathcal{U}$ are continuous and compact operators. Note that problem (1.1)-(1.2) has solutions if and only if the operator $\mathcal{T}$ has fixed points.

In the case $p>2$, we have $1<q<2$ due to $\frac{1}{p}+\frac{1}{q}=1$ and the following theorem is obtained.

Theorem 3.1 Let $p>2$ for each $i, j \in\{1,2\}, i \neq j, F_{i} \in C\left(\mathbb{N}_{\alpha_{1}+\beta_{1}-2, T+\alpha_{1}+\beta_{1}} \times \mathbb{N}_{\alpha_{2}+\beta_{2}-2, T+\alpha_{2}+\beta_{2}} \times\right.$ $\left.\mathbb{R}^{3}, \mathbb{R}\right), \varphi_{i} \in C\left(\mathbb{N}_{\alpha_{1}+\beta_{1}-2, T+\alpha_{1}+\beta_{1}} \times \mathbb{N}_{\alpha_{2}+\beta_{2}-2, T+\alpha_{2}+\beta_{2}},[0, \infty)\right)$ with $\varphi_{i}^{o}=\max \left\{\varphi\left(t_{i}-1, s\right)\right\}$. In addition, suppose that:

(H1) There exist constants $\chi_{i}>0$ and $0<\delta<\frac{1}{2-q}$ such that

$$
\chi_{i} \Delta_{C}^{\alpha}\left(t_{i}^{\alpha_{i}}\right)^{\delta} \leq F_{i}\left[t_{i}, t_{j}, x, y, z\right]
$$

for any $\left(t_{i}, t_{j}, x, y, z\right) \in \mathbb{N}_{\alpha_{i}+\beta_{i}-2, T+\alpha_{i}+\beta_{i}} \times \mathbb{N}_{\alpha_{j}+\beta_{j}-2, T+\alpha_{j}+\beta_{j}} \times \mathbb{R}^{3}$.

(H2) There exist constants $L_{i}, M_{i}, N_{i}>0$ such that, for each $t_{i} \in \mathbb{N}_{\alpha_{i}+\beta_{i}-2, T+\alpha_{i}+\beta_{i}}$ and $u_{1}, u_{2}, u_{3}, v_{1}, v_{2}, v_{3} \in \mathbb{R}$,

$$
\begin{aligned}
& \left|F_{i}\left[t_{i}, t_{j}, u_{1}, u_{2}, u_{3}\right]-F_{i}\left[t_{i}, t_{j}, v_{1}, v_{2}, v_{3}\right]\right| \\
& \quad \leq L_{i}\left|u_{1}-v_{1}\right|+M_{j}\left|u_{2}-v_{2}\right|+N_{j}\left|u_{3}-v_{3}\right| .
\end{aligned}
$$

(H3) $g_{i}<g_{i}\left(t_{i}\right)<G_{i}$ for each $t_{i} \in \mathbb{N}_{\alpha_{i}+\beta_{i}-2, T+\alpha_{i}+\beta_{i}}$.

Then problem (1.1)-(1.2) has a unique solution provided that

$$
\begin{aligned}
\Phi:= & \max \left\{\mathcal{K}_{1} \chi_{1}^{q-2} \Theta_{1}+\mathcal{K}_{2} \chi_{2}^{q-2} \Omega_{2}, \mathcal{K}_{1} \chi_{1}^{q-2} \frac{(T+2) \frac{-\gamma_{1}}{\Gamma\left(1-\gamma_{1}\right)}}{\Gamma} \Omega_{1}+\mathcal{K}_{2} \chi_{2}^{q-2} \frac{(T+2) \frac{-\gamma_{1}}{\Gamma\left(1-\gamma_{1}\right)}}{\Theta_{2}}\right. \\
& \left.\mathcal{K}_{1} \chi_{1}^{q-2} \Omega_{1}+\mathcal{K}_{2} \chi_{2}^{q-2} \Theta_{2}, \mathcal{K}_{1} \chi_{1}^{q-2} \frac{(T+2) \frac{-\gamma_{2}}{\Gamma\left(1-\gamma_{2}\right)}}{\Gamma} \Theta_{1}+\mathcal{K}_{2} \chi_{2}^{q-2} \frac{(T+2) \frac{-\gamma_{2}}{\Gamma\left(1-\gamma_{2}\right)}}{\Gamma} \Omega_{2}\right\} \\
< & 1,
\end{aligned}
$$


where

$$
\begin{aligned}
\mathcal{K}_{i}= & {\left[L_{i}+N_{j}+M_{j} \varphi_{j}^{o} \frac{\left(T+\omega_{j}+2\right)^{\omega_{j}}}{\Gamma\left(\omega_{j}+1\right)}\right] \frac{(q-1)}{\Gamma\left(\alpha_{i}+1\right)} } \\
\Omega_{i}= & {\left[1+\frac{\lambda_{i} G_{i}\left(\eta_{i}-\alpha_{i}-\beta_{i}+\theta_{i}+2\right)^{\theta_{i}}}{|\Lambda| \Gamma\left(\theta_{i}+1\right)}\right] \frac{1}{\Gamma\left(\beta_{i}\right)} \sum_{s=\alpha_{i}-1}^{T+\alpha_{i}}\left(T+\alpha_{i}+\beta_{i}-\sigma(s)\right)^{\beta_{i}-1}\left(s^{\underline{\alpha_{i}}}\right)^{\delta(q-2)+1} } \\
& +\frac{\lambda_{i} G_{i}\left(\eta_{i}-\alpha_{i}-\beta_{i}+\theta_{i}+1\right)^{\theta_{i}}}{|\Lambda| \Gamma\left(\theta_{i}+1\right)} \cdot \frac{1}{\Gamma\left(\beta_{i}\right)} \sum_{s=\alpha_{i}-1}^{\eta_{i}-\beta_{i}}\left(\eta_{i}-\sigma(s)\right)^{\frac{\beta_{i}-1}{}}\left(s^{\alpha_{i}}\right)^{\delta(q-2)+1} \\
\Theta_{i}= & \frac{1}{|\Lambda| \Gamma\left(\beta_{i}\right)} \sum_{s=\alpha_{i}-1}^{T+\alpha_{i}}\left(T+\alpha_{i}+\beta_{i}-\sigma(s)\right)^{\frac{\beta_{i}-1}{}}\left(s^{\alpha_{i}}\right)^{\delta(q-2)+1} \\
& +\frac{\lambda_{1} \lambda_{2} G_{1} G_{2}}{|\Lambda|} \cdot \frac{\left(\eta_{j}-\alpha_{j}-\beta_{j}+\theta_{j}+2\right)^{\frac{\theta_{j}}{2}}}{\Gamma\left(\theta_{j}+1\right)} \cdot \frac{\left(\eta_{i}-\alpha_{i}-\beta_{i}+\theta_{i}+1\right)^{\theta_{i}}}{\Gamma\left(\theta_{i}+1\right)} \\
& \times \frac{1}{\Gamma\left(\beta_{i}\right)} \sum_{s=\alpha_{i}-1}^{\eta_{i}-\beta_{i}}\left(\eta_{i}-\sigma(s)\right)^{\frac{\beta_{i}-1}{(s}}\left(s^{\alpha_{i}}\right)^{\delta(q-2)+1}
\end{aligned}
$$

Proof For each $i, j \in\{1,2\}, i \neq j$, by (H1) we have

$$
\chi_{i}\left(t_{i}^{\alpha_{i}}\right)^{\delta} \leq \frac{1}{\Gamma\left(\alpha_{i}\right)} \sum_{\xi=\alpha_{i}+\beta_{i}-1}^{t_{i}+\beta_{i}-1}\left(t_{i}+\alpha_{i}+\beta_{i}-1-\sigma(\xi)\right)^{\alpha_{i}-1} F_{i}^{*}\left(t_{j}, \xi\right) .
$$

By (A1), (H2), and the definition of operator $\mathcal{T}_{i}^{0}$, for any $\left(u_{1}, u_{2}\right),\left(v_{1}, v_{2}\right) \in \mathcal{C}$, we have

$$
\begin{aligned}
\mid & \left(\mathcal{T}_{i}^{0}\left(u_{1}, u_{2}\right)\right)\left(t_{1}, t_{2}\right)-\left(\mathcal{T}_{i}^{0}\left(v_{1}, v_{2}\right)\right)\left(t_{1}, t_{2}\right) \mid \\
= & \mid \phi_{q}\left[\sum_{\xi=\alpha_{i}+\beta_{i}-1}^{t_{i}+\beta_{i}-1} \frac{\left(t_{i}+\alpha_{i}+\beta_{i}-1-\sigma(\xi)\right)^{\alpha_{i}-1}}{\Gamma\left(\alpha_{i}\right)} F_{i}^{*}\left[u\left(t_{j}, \xi\right)\right]\right] \\
& -\phi_{q}\left[\sum_{\xi=\alpha_{i}+\beta_{i}-1}^{t_{i}+\beta_{i}-1} \frac{\left(t_{i}+\alpha_{i}+\beta_{i}-1-\sigma(\xi)\right)^{\alpha_{i}-1}}{\Gamma\left(\alpha_{i}\right)} F_{i}^{*}\left[v\left(t_{j}, \xi\right)\right] \mid\right. \\
\leq & (q-1)\left(\chi_{i}\left(t_{i}^{\alpha_{i}}\right)^{\delta}\right)^{q-2} \sum_{\xi=\alpha_{i}+\beta_{i}-1}^{t_{i}+\beta_{i}-1} \frac{\left(t_{i}+\alpha_{i}+\beta_{i}-1-\sigma(\xi)\right)^{\alpha_{i}-1}}{\Gamma\left(\alpha_{i}\right)}\left|F_{i}^{*}\left[u\left(t_{j}, \xi\right)\right]-F_{i}^{*}\left[v\left(t_{j}, \xi\right)\right]\right| \\
\leq & (q-1) \chi_{i}^{q-2} \frac{\left(t_{i}^{\frac{\alpha_{i}}{}}\right)^{\delta(q-2)+1}}{\Gamma\left(\alpha_{i}+1\right)}\left[L_{i}\left\|\Delta^{\gamma_{i}} u_{i}-\Delta^{\gamma_{i}} v_{i}\right\|+M_{j}\left\|\Psi^{\omega_{i}} u_{i}-\Psi^{\omega_{j}} v_{i}\right\|+N_{j}\left\|u_{j}-v_{j}\right\|\right] \\
\leq & (q-1) \chi_{i}^{q-2} \frac{\left(t_{i}^{\alpha_{i}}\right)^{\delta(q-2)+1}}{\Gamma\left(\alpha_{i}+1\right)}\left[L_{i}\left\|\Delta^{\gamma_{i}} u_{i}-\Delta^{\gamma_{i}} v_{i}\right\|\right. \\
& \left.+\left(N_{j}+M_{j} \varphi_{j}^{o} \frac{\left(T+\omega_{j}+2\right)^{\omega_{j}}}{\Gamma\left(\omega_{j}+1\right)}\right)\left\|u_{j}-v_{j}\right\|\right] \\
\leq & (q-1) \chi_{i}^{q-2} \frac{\left(t_{i}^{\frac{\alpha_{i}}{i}}\right)^{\delta(q-2)+1}}{\Gamma\left(\alpha_{i}+1\right)}\left[L_{i}+N_{j}+M_{j} \varphi_{j}^{o} \frac{\left(T+\omega_{j}+2\right)^{\omega_{j}}}{\Gamma\left(\omega_{j}+1\right)}\right] \\
& \times\left\|\left(u_{1}-v_{1}, u_{2}-v_{2}\right)\right\|
\end{aligned}
$$


Using (3.18) and (H3), we have

$$
\begin{aligned}
& \left|\mathcal{P}^{*}\left[F_{1}, F_{2}\right]\left(u_{1}, u_{2}\right)-\mathcal{P}^{*}\left[F_{1}, F_{2}\right]\left(v_{1}, v_{2}\right)\right| \\
& \leq \frac{1}{\Gamma\left(\beta_{1}\right)} \sum_{s=\alpha_{1}-1}^{T+\alpha_{1}}\left(T+\alpha_{1}+\beta_{1}-\sigma(s)\right) \frac{\beta_{1}-1}{}\left|\left(\mathcal{T}_{i}^{0}\left(u_{1}, u_{2}\right)\right)\left(s, t_{2}\right)-\left(\mathcal{T}_{i}^{0}\left(v_{1}, v_{2}\right)\right)\left(s, t_{2}\right)\right| \\
& -\frac{\lambda_{2}}{\Gamma\left(\beta_{2}\right) \Gamma\left(\theta_{2}\right)} \sum_{r=\alpha_{2}+\beta_{2}-1}^{\eta_{2}} \sum_{s=\alpha_{2}-1}^{r-\beta_{2}}\left(\eta_{2}+\theta_{2}-\sigma(r)\right)^{\frac{\theta_{2}-1}{}}(r-\sigma(s))^{\frac{\beta_{2}-1}{g_{2}}} g_{2}(r) \\
& \times\left|\left(\mathcal{T}_{i}^{0}\left(u_{1}, u_{2}\right)\right)\left(t_{1}, s\right)-\left(\mathcal{T}_{i}^{0}\left(v_{1}, v_{2}\right)\right)\left(t_{1}, s\right)\right| \\
& \leq \frac{\chi_{1}^{q-2} \mathcal{K}_{1}}{\Gamma\left(\beta_{1}\right)}\left\|\left(u_{1}-v_{1}, u_{2}-v_{2}\right)\right\|_{\mathcal{C}_{1}} \sum_{s=\alpha_{1}-1}^{T+\alpha_{1}}\left(T+\alpha_{1}+\beta_{1}-\sigma(s)\right)^{\frac{\beta_{1}-1}{}\left(s \frac{\alpha_{1}}{\delta(q-2)+1}\right.} \\
& -\frac{\lambda_{2} G_{2} \chi_{2}^{q-2} \mathcal{K}_{2}}{\Gamma\left(\beta_{2}\right) \Gamma\left(\theta_{2}\right)}\left\|\left(u_{1}-v_{1}, u_{2}-v_{2}\right)\right\|_{\mathcal{C}_{2}} \sum_{r=\alpha_{2}+\beta_{2}-1}^{\eta_{2}} \sum_{s=\alpha_{2}-1}^{r-\beta_{2}}\left(\eta_{2}+\theta_{2}-\sigma(r)\right) \frac{\theta_{2}-1}{} \\
& \times(r-\sigma(s))^{\beta_{2}-1}\left(s^{\underline{\alpha_{2}}}\right)^{\delta(q-2)+1},
\end{aligned}
$$

and

$$
\begin{aligned}
& \left|\mathcal{Q}^{*}\left[F_{1}, F_{2}\right]\left(u_{1}, u_{2}\right)-\mathcal{Q}^{*}\left[F_{1}, F_{2}\right]\left(v_{1}, v_{2}\right)\right| \\
& =\frac{\chi_{2}^{q-2} \mathcal{K}_{2}}{\Gamma\left(\beta_{2}\right)}\left\|\left(u_{1}-v_{1}, u_{2}-v_{2}\right)\right\|_{\mathcal{C}_{2}} \sum_{s=\alpha_{2}-1}^{T+\alpha_{2}}\left(T+\alpha_{2}+\beta_{2}-\sigma(s)\right)^{\frac{\beta_{2}-1}{}\left(s \frac{\alpha_{2}}{}\right)^{\delta(q-2)+1}} \\
& \quad-\frac{\lambda_{1} G_{1} \chi_{1}^{q-2} \mathcal{K}_{1}}{\Gamma\left(\beta_{1}\right) \Gamma\left(\theta_{1}\right)}\left\|\left(u_{1}-v_{1}, u_{2}-v_{2}\right)\right\|_{\mathcal{C}_{1}} \sum_{r=\alpha_{1}+\beta_{1}-1}^{\eta_{1}} \sum_{s=\alpha_{1}-1}^{r-\beta_{1}}\left(\eta_{1}+\theta_{1}-\sigma(r)\right)^{\frac{\theta_{1}-1}{}} \\
& \quad \times(r-\sigma(s))^{\frac{\beta_{1}-1}{(}\left(s \frac{\alpha_{1}}{}\right)^{\delta(q-2)+1} .}
\end{aligned}
$$

From (3.19)-(3.20), it implies that

$$
\begin{aligned}
\left|\left(\mathcal{T}_{1}\left(u_{1}, u_{2}\right)\right)\left(t_{1}, t_{2}\right)-\left(\mathcal{T}_{1}\left(v_{1}, v_{2}\right)\right)\left(t_{1}, t_{2}\right)\right| \\
=\left|\left(\mathcal{T}_{1}^{*}\left(\mathcal{T}_{1}^{o}\left(u_{1}, u_{2}\right)\right)\right)\left(t_{1}, t_{2}\right)-\left(\mathcal{T}_{1}^{*}\left(\mathcal{T}_{1}^{o}\left(v_{1}, v_{2}\right)\right)\right)\left(t_{1}, t_{2}\right)\right| \\
\leq \frac{1}{\Gamma\left(\beta_{1}\right)} \sum_{s=\alpha_{1}-1}^{t_{1}-\beta_{1}}\left(t_{1}-\sigma(s)\right) \frac{\beta_{1}-1}{}\left|\left(\mathcal{T}_{1}^{0}\left(u_{1}, u_{2}\right)\right)\left(t_{1}, t_{2}\right)-\left(\mathcal{T}_{1}^{0}\left(v_{1}, v_{2}\right)\right)\left(t_{1}, t_{2}\right)\right| \\
\quad+\frac{1}{|\Lambda|}\left\{\left(\frac{\lambda_{1}}{\Gamma\left(\theta_{1}\right)} \sum_{s=\alpha_{1}+\beta_{1}-2}^{\eta_{1}}\left(\eta_{1}+\theta_{1}-\sigma(s)\right)^{\left.\frac{\theta_{1}-1}{} g_{1}(s)\right)}\right.\right. \\
\quad \times\left|\mathcal{P}^{*}\left[F_{1}, F_{2}\right]\left(u_{1}, u_{2}\right)-\mathcal{P}^{*}\left[F_{1}, F_{2}\right]\left(v_{1}, v_{2}\right)\right| \\
\left.\quad+\left|\mathcal{Q}^{*}\left[F_{1}, F_{2}\right]\left(u_{1}, u_{2}\right)-\mathcal{Q}^{*}\left[F_{1}, F_{2}\right]\left(v_{1}, v_{2}\right)\right|\right\} \\
\leq \\
\quad \frac{\chi_{1}^{q-2} \mathcal{K}_{1}\left\|\left(u_{1}-v_{1}, u_{2}-v_{2}\right)\right\| \mathcal{C}_{1}}{\Gamma\left(\beta_{1}\right)} \sum_{s=\alpha_{1}-1}^{t_{1}-\beta_{1}}\left(t_{1}-\sigma(s)\right)^{\frac{\beta_{1}-1}{\left(s \frac{\alpha_{1}}{\delta(q-2)+1}\right.}}
\end{aligned}
$$




$$
\begin{aligned}
& +\frac{1}{|\Lambda|}\left\{\frac{\lambda_{1} G_{1}\left(\eta_{1}-\alpha_{1}-\beta_{1}+\theta_{1}+2\right)^{\theta_{1}}}{|\Lambda| \Gamma\left(\theta_{1}+1\right)}\right. \\
& \times\left[\frac{\chi_{1}^{q-2} \mathcal{K}_{1}}{\Gamma\left(\beta_{1}\right)}\left\|\left(u_{1}-v_{1}, u_{2}-v_{2}\right)\right\|_{\mathcal{C}_{1}} \sum_{s=\alpha_{1}-1}^{T+\alpha_{1}}\left(T+\alpha_{1}+\beta_{1}-\sigma(s)\right)^{\frac{\beta_{1}-1}{}}\left(s \frac{\alpha_{1}}{}\right)^{\delta(q-2)+1}\right. \\
& +\frac{\lambda_{2} G_{2} \chi_{2}^{q-2} \mathcal{K}_{2}}{\Gamma\left(\beta_{2}\right) \Gamma\left(\theta_{2}\right)}\left\|\left(u_{1}-v_{1}, u_{2}-v_{2}\right)\right\|_{\mathcal{C}_{2}} \sum_{r=\alpha_{2}+\beta_{2}-1}^{\eta_{2}} \sum_{s=\alpha_{2}-1}^{r-\beta_{2}}\left(\eta_{2}+\theta_{2}-\sigma(r)\right)^{\theta_{2}-1} \\
& \left.\times(r-\sigma(s))^{\frac{\beta_{2}-1}{}}\left(s^{\alpha_{2}}\right)^{\delta(q-2)+1}\right] \\
& +\left[\frac{\chi_{2}^{q-2} \mathcal{K}_{2}}{\Gamma\left(\beta_{2}\right)}\left\|\left(u_{1}-v_{1}, u_{2}-v_{2}\right)\right\|_{\mathcal{C}_{2}} \sum_{s=\alpha_{2}-1}^{T+\alpha_{2}}\left(T+\alpha_{2}+\beta_{2}-\sigma(s)\right)^{\frac{\beta_{2}-1}{}}\left(s^{\alpha_{2}}\right)^{\delta(q-2)+1}\right. \\
& +\frac{\lambda_{1} G_{1} \chi_{1}^{q-2} \mathcal{K}_{1}}{\Gamma\left(\beta_{1}\right) \Gamma\left(\theta_{1}\right)}\left\|\left(u_{1}-v_{1}, u_{2}-v_{2}\right)\right\|_{\mathcal{C}_{1}} \sum_{r=\alpha_{1}+\beta_{1}-1}^{\eta_{1}} \sum_{s=\alpha_{1}-1}^{r-\beta_{1}}\left(\eta_{1}+\theta_{1}-\sigma(r)\right) \frac{\theta_{1}-1}{\underline{n}} \\
& \left.\left.\times(r-\sigma(s))^{\frac{\beta_{1}-1}{}}\left(s^{\underline{\alpha_{1}}}\right)^{\delta(q-2)+1}\right]\right\} \\
& \leq\left\|\left(u_{1}-v_{1}, u_{2}-v_{2}\right)\right\|_{\mathcal{U}}\left\{\mathcal{K}_{1} \chi_{1}^{q-2} \Omega_{1}+\mathcal{K}_{2} \chi_{2}^{q-2} \Theta_{2}\right\} .
\end{aligned}
$$

Similarly, we can find that

$$
\begin{aligned}
& \left|\left(\mathcal{T}_{2}\left(u_{1}, u_{2}\right)\right)\left(t_{1}, t_{2}\right)-\left(\mathcal{T}_{2}\left(v_{1}, v_{2}\right)\right)\left(t_{1}, t_{2}\right)\right| \\
& \quad \leq\left\|\left(u_{1}-v_{1}, u_{2}-v_{2}\right)\right\|_{\mathcal{U}}\left\{\mathcal{K}_{1} \chi_{1}^{q-2} \Theta_{1}+\mathcal{K}_{2} \chi_{2}^{q-2} \Omega_{2}\right\} .
\end{aligned}
$$

Next, taking the fractional difference of order $\gamma_{1}, \gamma_{2}$ for (3.2) and (3.3), respectively, we obtain

$$
\begin{aligned}
& \Delta^{\gamma_{1}}\left(\mathcal{T}_{1}\left(u_{1}, u_{2}\right)\right)\left(t_{1}, t_{2}\right) \\
& =\Delta^{\gamma_{1}}\left(\mathcal{T}_{1}^{*}\left(\mathcal{T}_{1}^{o}\left(u_{1}, u_{2}\right)\right)\right)\left(t_{1}, t_{2}\right) \\
& =\frac{1}{\Gamma\left(-\gamma_{1}\right) \Gamma\left(\beta_{1}\right)} \sum_{r=\alpha_{1}+\beta_{1}-1}^{t_{1}+\gamma_{1}} \sum_{s=\alpha_{1}-1}^{r-\beta_{1}}\left(t_{1}-\sigma(r)\right) \frac{-\gamma_{1}-1}{}(r-\sigma(s))^{\frac{\beta_{1}-1}{-}}\left(\mathcal{T}_{1}^{o}\left(u_{1}, u_{2}\right)\right)\left(t_{2}, s\right) \\
& +\frac{1}{\Lambda}\left\{\frac{\lambda_{1}}{\Gamma\left(\theta_{1}\right) \Gamma\left(-\gamma_{1}\right)} \sum_{r=\alpha_{1}+\beta_{1}-1}^{t_{1}+\gamma_{1}} \sum_{s=\alpha_{1}+\beta_{1}-2}^{\eta_{1}}\left(t_{1}-\sigma(r)\right) \frac{-\gamma_{1}-1}{(}\left(\eta_{1}+\theta_{1}-\sigma(s)\right) \frac{\theta_{1}-1}{g_{1}}(s)\right. \\
& \left.\times \mathcal{P}^{*}\left[F_{1}, F_{2}\right]\left(u_{1}, u_{2}\right)+\frac{1}{\Gamma\left(-\gamma_{1}\right)} \sum_{s=\alpha_{1}+\beta_{1}-1}^{t_{1}+\gamma_{1}}\left(t_{1}-\sigma(s)\right)^{-\frac{\gamma_{1}-1}{}} \mathcal{Q}^{*}\left[F_{1}, F_{2}\right]\left(u_{1}, u_{2}\right)\right\}
\end{aligned}
$$

and

$$
\begin{aligned}
& \Delta^{\gamma_{2}}\left(\mathcal{T}_{2}\left(u_{1}, u_{2}\right)\right)\left(t_{1}, t_{2}\right) \\
& \quad=\Delta^{\gamma_{2}}\left(\mathcal{T}_{2}^{*}\left(\mathcal{T}_{2}^{o}\left(u_{1}, u_{2}\right)\right)\right)\left(t_{1}, t_{2}\right)
\end{aligned}
$$




$$
\begin{aligned}
= & \frac{1}{\Gamma\left(-\gamma_{2}\right) \Gamma\left(\beta_{2}\right)} \sum_{r=\alpha_{2}+\beta_{2}-1}^{t_{2}+\gamma_{2}} \sum_{s=\alpha_{2}-1}^{r-\beta_{2}}\left(t_{2}-\sigma(r)\right)^{\frac{-\gamma_{2}-1}{}}(r-\sigma(s))^{\frac{\beta_{2}-1}{}}\left(\mathcal{T}_{2}^{o}\left(u_{1}, u_{2}\right)\right)\left(t_{1}, s\right) \\
& +\frac{1}{\Lambda}\left\{\frac{\lambda_{2}}{\Gamma\left(-\gamma_{2}\right) \Gamma\left(\theta_{2}\right)} \sum_{r=\alpha_{2}+\beta_{2}-1}^{t_{2}+\gamma_{2}} \sum_{s=\alpha_{2}+\beta_{2}-2}^{\eta_{2}}\left(t_{2}-\sigma(r)\right)^{\frac{-\gamma_{2}-1}{}}\left(\eta_{2}+\theta_{2}-\sigma(s)\right)^{\frac{\theta_{1}-1}{g_{2}}} g_{2}(s)\right. \\
& \left.\times \mathcal{Q}^{*}\left[F_{1}, F_{2}\right]\left(u_{1}, u_{2}\right)+\frac{1}{\Gamma\left(-\gamma_{2}\right)} \sum_{s=\alpha_{2}+\beta_{2}-1}^{t_{2}+\gamma_{2}}\left(t_{2}-\sigma(s)\right)^{\frac{-\gamma_{2}-1}{2}} \mathcal{P}^{*}\left[F_{1}, F_{2}\right]\left(u_{1}, u_{2}\right)\right\},(3
\end{aligned}
$$

where $t_{i} \in \mathbb{N}_{\alpha_{i}+\beta_{i}-\gamma_{i}+1, T+\alpha_{i}+\beta_{i}-\gamma_{i}}$. Therefore,

$$
\begin{aligned}
& \left|\Delta^{\gamma_{1}}\left(\mathcal{T}_{1}\left(u_{1}, u_{2}\right)\right)\left(t_{1}, t_{2}\right)-\Delta^{\gamma_{1}}\left(\mathcal{T}_{1}\left(v_{1}, v_{2}\right)\right)\left(t_{1}, t_{2}\right)\right| \\
& \leq \frac{1}{\Gamma\left(-\gamma_{1}\right) \Gamma\left(\beta_{1}\right)} \sum_{r=\alpha_{1}+\beta_{1}-1}^{t_{1}+\gamma_{1}} \sum_{s=\alpha_{1}-1}^{r-\beta_{1}}\left(t_{1}-\sigma(r)\right)^{-\frac{\gamma_{1}-1}{}}(r-\sigma(s))^{\frac{\beta_{1}-1}{2}} \\
& \times\left|\left(\mathcal{T}_{1}^{*}\left(\mathcal{T}_{1}^{o}\left(u_{1}, u_{2}\right)\right)\right)\left(t_{1}, t_{2}\right)-\left(\mathcal{T}_{1}^{*}\left(\mathcal{T}_{1}^{o}\left(v_{1}, v_{2}\right)\right)\right)\left(t_{1}, t_{2}\right)\right| \\
& +\frac{1}{\Lambda}\left\{\frac{\lambda_{1} G_{1}}{\Gamma\left(\theta_{1}\right) \Gamma\left(-\gamma_{1}\right)} \sum_{x=\alpha_{1}+\beta_{1}-1}^{t_{1}+\gamma_{1}} \sum_{y=\alpha_{1}+\beta_{1}-2}^{\eta_{1}}\left(t_{1}-\sigma(x)\right) \frac{-\gamma_{1}-1}{n}\left(\eta_{1}+\theta_{1}-\sigma(y)\right)^{\theta_{1}-1}\right. \\
& \times\left|\mathcal{P}^{*}\left[F_{1}, F_{2}\right]\left(u_{1}, u_{2}\right)-\mathcal{P}^{*}\left[F_{1}, F_{2}\right]\left(v_{1}, v_{2}\right)\right| \\
& \left.+\frac{1}{\Gamma\left(-\gamma_{1}\right)} \sum_{x=\alpha_{1}+\beta_{1}-1}^{t_{1}+\gamma_{1}}\left(t_{1}-\sigma(x)\right) \frac{-\gamma_{1}-1}{-}\left|\mathcal{Q}^{*}\left[F_{1}, F_{2}\right]\left(u_{1}, u_{2}\right)-\mathcal{Q}^{*}\left[F_{1}, F_{2}\right]\left(v_{1}, v_{2}\right)\right|\right\} \\
& \leq \frac{\chi_{1}^{q-2} \mathcal{K}_{1}\left\|\left(u_{1}-v_{1}, u_{2}-v_{2}\right)\right\|_{\mathcal{C}_{1}}}{\Gamma\left(-\gamma_{1}\right) \Gamma\left(\beta_{1}\right)} \cdot \frac{(T+2) \frac{-\gamma_{1}}{\Gamma\left(1-\gamma_{1}\right)}}{\sum_{s=\alpha_{1}-1}^{T+\alpha_{1}}}\left(T+\alpha_{1}+\beta_{1}-\sigma(s)\right)^{\frac{\beta_{1}-1}{}}\left(s^{\alpha_{1}}\right)^{\delta(q-2)+1} \\
& +\frac{1}{\Lambda}\left\{\lambda_{1} G_{1} \cdot \frac{(T+2) \frac{-\gamma_{1}}{\Gamma\left(1-\gamma_{1}\right)}}{\Gamma} \cdot \frac{\left(\eta_{1}-\alpha_{1}-\beta_{1}+\theta_{1}+2\right)^{\theta_{1}}}{\Gamma\left(\theta_{1}+1\right)}\right. \\
& \times\left[\frac{\chi_{1}^{q-2} \mathcal{K}_{1}}{\Gamma\left(\beta_{1}\right)}\left\|\left(u_{1}-v_{1}, u_{2}-v_{2}\right)\right\|_{\mathcal{C}_{1}} \sum_{s=\alpha_{1}-1}^{T+\alpha_{1}}\left(T+\alpha_{1}+\beta_{1}-\sigma(s)\right)^{\beta_{1}-1}\left(s^{\frac{\alpha_{1}}{}}\right)^{\delta(q-2)+1}\right. \\
& +\frac{\lambda_{2} G_{2} \chi_{2}^{q-2} \mathcal{K}_{2}}{\Gamma\left(\beta_{2}\right) \Gamma\left(\theta_{2}\right)}\left\|\left(u_{1}-v_{1}, u_{2}-v_{2}\right)\right\|_{\mathcal{C}_{2}} \sum_{r=\alpha_{2}+\beta_{2}-1}^{\eta_{2}} \sum_{s=\alpha_{2}-1}^{r-\beta_{2}}\left(\eta_{2}+\theta_{2}-\sigma(r)\right)^{\theta_{2}-1} \\
& \left.\times(r-\sigma(s))^{\beta_{2}-1}\left(s^{\frac{\alpha_{2}}{}}\right)^{\delta(q-2)+1}\right]+\frac{(T+2) \underline{-\gamma_{1}}}{\Gamma\left(1-\gamma_{1}\right)} \\
& \times\left[\frac{\chi_{2}^{q-2} \mathcal{K}_{2}}{\Gamma\left(\beta_{2}\right)}\left\|\left(u_{1}-v_{1}, u_{2}-v_{2}\right)\right\|_{\mathcal{C}_{2}} \sum_{s=\alpha_{2}-1}^{T+\alpha_{2}}\left(T+\alpha_{2}+\beta_{2}-\sigma(s)\right)^{\beta_{2}-1}\left(s^{\frac{\alpha_{2}}{2}}\right)^{\delta(q-2)+1}\right. \\
& +\frac{\lambda_{1} G_{1} \chi_{1}^{q-2} \mathcal{K}_{1}}{\Gamma\left(\beta_{1}\right) \Gamma\left(\theta_{1}\right)}\left\|\left(u_{1}-v_{1}, u_{2}-v_{2}\right)\right\|_{\mathcal{C}_{1}} \sum_{r=\alpha_{1}+\beta_{1}-1}^{\eta_{1}} \sum_{s=\alpha_{1}-1}^{r-\beta_{1}}\left(\eta_{1}+\theta_{1}-\sigma(r)\right)^{\frac{\theta_{1}-1}{}} \\
& \left.\left.\times(r-\sigma(s))^{\beta_{1}-1}\left(s^{\alpha_{1}}\right)^{\delta(q-2)+1}\right]\right\} \\
& \leq\left\|\left(u_{1}-v_{1}, u_{2}-v_{2}\right)\right\|_{\mathcal{U}}\left\{\mathcal{K}_{1} \chi_{1}^{q-2} \frac{(T+2) \frac{-\gamma_{1}}{\Gamma}}{\Gamma\left(1-\gamma_{1}\right)} \Omega_{1}+\mathcal{K}_{2} \chi_{2}^{q-2} \frac{(T+2) \frac{-\gamma_{1}}{\Gamma\left(1-\gamma_{1}\right)}}{\Gamma} \Theta_{2}\right\} .
\end{aligned}
$$


Similarly, we obtain

$$
\begin{aligned}
& \left|\Delta^{\gamma_{2}}\left(\mathcal{T}_{2}\left(u_{1}, u_{2}\right)\right)\left(t_{1}, t_{2}\right)-\Delta^{\gamma_{2}}\left(\mathcal{T}_{2}\left(v_{1}, v_{2}\right)\right)\left(t_{1}, t_{2}\right)\right| \\
& \quad \leq\left\|\left(u_{1}-v_{1}, u_{2}-v_{2}\right)\right\|_{\mathcal{U}}\left\{\mathcal{K}_{1} \chi_{1}^{q-2} \frac{(T+2) \frac{-\gamma_{1}}{\Gamma\left(1-\gamma_{1}\right)}}{\Gamma} \Theta_{1}+\mathcal{K}_{2} \chi_{2}^{q-2} \frac{(T+2) \frac{-\gamma_{1}}{\Gamma\left(1-\gamma_{1}\right)}}{\Gamma} \Omega_{2}\right\} .
\end{aligned}
$$

From (3.22) and (3.25), we find that

$$
\begin{aligned}
& \left\|\left(\mathcal{T}\left(u_{1}, u_{2}\right)\right)-\left(\mathcal{T}\left(v_{1}, v_{2}\right)\right)\right\|_{\mathcal{C}_{1}} \\
& <\left\|\left(u_{1}-v_{1}, u_{2}-v_{2}\right)\right\|_{\mathcal{U}} \\
& \quad \times \max \left\{\mathcal{K}_{1} \chi_{1}^{q-2} \Theta_{1}+\mathcal{K}_{2} \chi_{2}^{q-2} \Omega_{2}, \mathcal{K}_{1} \chi_{1}^{q-2} \frac{(T+2) \frac{-\gamma_{1}}{\Gamma\left(1-\gamma_{1}\right)}}{\Gamma} \Omega_{1}+\mathcal{K}_{2} \chi_{2}^{q-2} \frac{(T+2) \frac{-\gamma_{1}}{\Gamma\left(1-\gamma_{1}\right)}}{\Theta_{2}}\right\} .
\end{aligned}
$$

In addition, by (3.21) and (3.26), we find that

$$
\begin{aligned}
& \left\|\left(\mathcal{T}\left(u_{1}, u_{2}\right)\right)-\left(\mathcal{T}\left(v_{1}, v_{2}\right)\right)\right\|_{\mathcal{C}_{2}} \\
& <\left\|\left(u_{1}-v_{1}, u_{2}-v_{2}\right)\right\|_{\mathcal{U}} \\
& \times \max \left\{\mathcal{K}_{1} \chi_{1}^{q-2} \Omega_{1}+\mathcal{K}_{2} \chi_{2}^{q-2} \Theta_{2}, \mathcal{K}_{1} \chi_{1}^{q-2} \frac{(T+2) \frac{-\gamma_{2}}{\Gamma\left(1-\gamma_{2}\right)}}{\Gamma} \Theta_{1}+\mathcal{K}_{2} \chi_{2}^{q-2} \frac{(T+2) \frac{-\gamma_{2}}{\Gamma\left(1-\gamma_{2}\right)}}{\Gamma 2}\right\} .
\end{aligned}
$$

Hence, from (3.27) and (3.28), we can conclude that

$$
\begin{aligned}
\| & \left(\mathcal{T}\left(u_{1}, u_{2}\right)\right)-\left(\mathcal{T}\left(v_{1}, v_{2}\right)\right) \|_{\mathcal{U}} \\
< & \left\|\left(u_{1}-v_{1}, u_{2}-v_{2}\right)\right\|_{\mathcal{U}} \\
& \times \max \left\{\mathcal{K}_{1} \chi_{1}^{q-2} \Theta_{1}+\mathcal{K}_{2} \chi_{2}^{q-2} \Omega_{2}, \mathcal{K}_{1} \chi_{1}^{q-2} \frac{(T+2) \frac{-\gamma_{1}}{\Gamma\left(1-\gamma_{1}\right)}}{\Gamma} \Omega_{1}+\mathcal{K}_{2} \chi_{2}^{q-2} \frac{(T+2) \frac{-\gamma_{1}}{\Gamma\left(1-\gamma_{1}\right)}}{\Theta_{2}},\right. \\
& \left.\mathcal{K}_{1} \chi_{1}^{q-2} \Omega_{1}+\mathcal{K}_{2} \chi_{2}^{q-2} \Theta_{2}, \mathcal{K}_{1} \chi_{1}^{q-2} \frac{(T+2) \frac{-\gamma_{2}}{\Gamma\left(1-\gamma_{2}\right)}}{\Gamma} \Theta_{1}+\mathcal{K}_{2} \chi_{2}^{q-2} \frac{(T+2) \frac{-\gamma_{2}}{\Gamma\left(1-\gamma_{2}\right)}}{\Gamma} \Omega_{2}\right\} \\
= & \left\|\left(u_{1}-v_{1}, u_{2}-v_{2}\right)\right\|_{\mathcal{U}} \Phi .
\end{aligned}
$$

By (3.13), $\mathcal{T}$ is a contraction mapping. Hence, by the Banach fixed point theorem, we get that $\mathcal{T}$ has a fixed point, which is a unique solution of problem (1.1)-(1.2).

In the same manner as Theorem 3.1, we can obtain the following theorem.

Theorem 3.2 Let $p>2,(\mathrm{H} 2)-(\mathrm{H} 3)$ hold, and the following condition hold:

(H4) There exist constants $\chi_{i}>0$ and $0<\delta<\frac{1}{2-q}$ such that

$$
F_{i}\left[t_{i}, t_{j}, x, y, z\right] \leq-\chi_{i} \Delta_{C}^{\alpha}\left(t_{i}^{\alpha_{i}}\right)^{\delta}
$$

$$
\text { for any }\left(t_{i}, t_{j}, x, y, z\right) \in \mathbb{N}_{\alpha_{i}+\beta_{i}-2, T+\alpha_{i}+\beta_{i}} \times \mathbb{N}_{\alpha_{j}+\beta_{j}-2, T+\alpha_{j}+\beta_{j}} \times \mathbb{R}^{3} .
$$

Then problem (1.1)-(1.2) has a unique solution. 
In the case $1<p<2$ and $q>2$ since $\frac{1}{p}+\frac{1}{q}=1$, we obtain the following theorem.

Theorem 3.3 Let $1<p<2$ and $(\mathrm{H} 2)-(\mathrm{H} 3)$ hold. For each $i, j \in\{1,2\}, i \neq j, F_{i} \in$ $C\left(\mathbb{N}_{\alpha_{1}+\beta_{1}-2, T+\alpha_{1}+\beta_{1}} \times \mathbb{N}_{\alpha_{2}+\beta_{2}-2, T+\alpha_{2}+\beta_{2}} \times \mathbb{R}^{3}, \mathbb{R}\right), \varphi_{i} \in C\left(\mathbb{N}_{\alpha_{1}+\beta_{1}-2, T+\alpha_{1}+\beta_{1}} \times \mathbb{N}_{\alpha_{2}+\beta_{2}-2, T+\alpha_{2}+\beta_{2}}\right.$, $[0, \infty))$ with $\varphi_{i}^{o}=\max \left\{\varphi\left(t_{i}-1, s\right)\right\}$. Suppose that the following assumption holds:

(H5) There exists a nonnegative function $k_{i} \in C\left(\mathbb{N}_{\alpha_{1}+\beta_{1}-2, T+\alpha_{1}+\beta_{1}} \times \mathbb{N}_{\alpha_{2}+\beta_{2}-2, T+\alpha_{2}+\beta_{2}}\right.$, $[0, \infty))$ and $\mathcal{M}_{i}:=\frac{1}{\Gamma\left(\alpha_{i}\right)} \sum_{\xi=\alpha_{i}+\beta_{i}-1}^{T+\alpha_{i}+\beta_{i}-1}\left(T+2 \alpha_{i}+\beta_{i}-1-\sigma(\xi)\right)^{\alpha_{i}-1} k_{i}\left(T+\alpha_{j}+\beta_{j}, \xi\right)>0$ such that

$$
F_{i}\left[t_{i}, t_{j}, x, y, z\right] \leq k_{i}\left(t_{i}, t_{j}\right)
$$

for any $\left(t_{i}, t_{j}, x, y, z\right) \in \mathbb{N}_{\alpha_{i}+\beta_{i}-2, T+\alpha_{i}+\beta_{i}} \times \mathbb{N}_{\alpha_{j}+\beta_{j}-2, T+\alpha_{j}+\beta_{j}} \times \mathbb{R}^{3}$.

Then problem (1.1)-(1.2) has a unique solution provided that

$$
\begin{aligned}
\Upsilon: & \max \left\{\mathcal{K}_{1} \mathcal{M}_{1}^{q-2} \bar{\Theta}_{1}+\mathcal{K}_{2} \mathcal{M}_{2}^{q-2} \bar{\Omega}_{2}, \mathcal{K}_{1} \mathcal{M}_{1}^{q-2} \frac{(T+2) \frac{-\gamma_{1}}{\Gamma\left(1-\gamma_{1}\right)}}{\Omega_{1}}+\mathcal{K}_{2} \mathcal{M}_{2}^{q-2} \frac{(T+2) \frac{-\gamma_{1}}{\Gamma\left(1-\gamma_{1}\right)}}{\bar{\Theta}_{2}},\right. \\
& \mathcal{K}_{1} \mathcal{M}_{1}^{q-2} \bar{\Omega}_{1}+\mathcal{K}_{2} \mathcal{M}_{2}^{q-2} \bar{\Theta}_{2}, \mathcal{K}_{1} \mathcal{M}_{1}^{q-2} \frac{(T+2) \frac{-\gamma_{2}}{\Gamma\left(1-\gamma_{2}\right)}}{\Gamma} \bar{\Theta}_{1}+\mathcal{K}_{2} \mathcal{M}_{2}^{q-2} \frac{(T+2) \frac{-\gamma_{2}}{\Gamma\left(1-\gamma_{2}\right)}}{\left.\Omega_{2}\right\}} \\
< & 1,
\end{aligned}
$$

where $\mathcal{K}_{i}$ is defined as (3.14), and

$$
\begin{aligned}
\bar{\Omega}_{i}= & {\left[1+\frac{\lambda_{i} G_{i}\left(\eta_{i}-\alpha_{i}-\beta_{i}+\theta_{i}+2\right) \underline{\theta_{i}}}{|\Lambda| \Gamma\left(\theta_{i}+1\right)}\right] \frac{1}{\Gamma\left(\beta_{i}\right)} \sum_{s=\alpha_{i}-1}^{T+\alpha_{i}}\left(T+\alpha_{i}+\beta_{i}-\sigma(s)\right)^{\frac{\beta_{i}-1}{}} s \underline{\alpha_{i}} } \\
& +\frac{\lambda_{i} G_{i}\left(\eta_{i}-\alpha_{i}-\beta_{i}+\theta_{i}+1\right) \underline{\theta_{i}}}{|\Lambda| \Gamma\left(\theta_{i}+1\right)} \cdot \frac{1}{\Gamma\left(\beta_{i}\right)} \sum_{s=\alpha_{i}-1}^{\eta_{i}-\beta_{i}}\left(\eta_{i}-\sigma(s)\right)^{\frac{\beta_{i}-1}{}} s \underline{\alpha_{i}} \\
\bar{\Theta}_{i}= & \frac{1}{|\Lambda| \Gamma\left(\beta_{i}\right)} \sum_{s=\alpha_{i}-1}^{T+\alpha_{i}}\left(T+\alpha_{i}+\beta_{i}-\sigma(s)\right)^{\frac{\beta_{i}-1}{}} s^{\alpha_{i}} \\
& +\frac{\lambda_{1} \lambda_{2} G_{1} G_{2}}{|\Lambda|} \cdot \frac{\left(\eta_{j}-\alpha_{j}-\beta_{j}+\theta_{j}+2\right)^{\frac{\theta_{j}}{}}}{\Gamma\left(\theta_{j}+1\right)} \cdot \frac{\left(\eta_{i}-\alpha_{i}-\beta_{i}+\theta_{i}+1\right)^{\theta_{i}}}{\Gamma\left(\theta_{i}+1\right)} \\
& \times \frac{1}{\Gamma\left(\beta_{i}\right)} \sum_{s=\alpha_{i}-1}^{\eta_{i}-\beta_{i}}\left(\eta_{i}-\sigma(s)\right)^{\frac{\beta_{i}-1}{s}} s \underline{\alpha_{i}} .
\end{aligned}
$$

Proof For each $i, j \in\{1,2\}, i \neq j$, by (H5) we have

$$
\begin{aligned}
& \left|\frac{1}{\Gamma\left(\alpha_{i}\right)} \sum_{\xi=\alpha_{i}+\beta_{i}-1}^{t_{i}+\beta_{i}-1}\left(t_{i}+\alpha_{i}+\beta_{i}-1-\sigma(\xi)\right) \frac{\alpha_{i}-1}{} F_{i}^{*}\left[u\left(t_{j}, \xi\right)\right]\right| \\
& \quad \leq \frac{1}{\Gamma\left(\alpha_{i}\right)} \sum_{\xi=\alpha_{i}+\beta_{i}-1}^{T+\alpha_{i}+\beta_{i}-1}\left(T+2 \alpha_{i}+\beta_{i}-1-\sigma(\xi)\right) \frac{\alpha_{i}-1}{} k_{i}\left(t_{j}, \xi\right) \\
& \quad \leq \mathcal{M}_{i} .
\end{aligned}
$$


By (A2), (H2), and the definition of operator $\mathcal{T}_{i}^{0}$, for any $\left(u_{1}, u_{2}\right),\left(v_{1}, v_{2}\right) \in \mathcal{C}$, we have

$$
\begin{aligned}
\mid & \left(\mathcal{T}_{i}^{0}\left(u_{1}, u_{2}\right)\right)\left(t_{1}, t_{2}\right)-\left(\mathcal{T}_{i}^{0}\left(v_{1}, v_{2}\right)\right)\left(t_{1}, t_{2}\right) \mid \\
= & \mid \phi_{q}\left[\sum_{\xi=\alpha_{i}+\beta_{i}-1}^{t_{i}+\beta_{i}-1} \frac{\left(t_{i}+\alpha_{i}+\beta_{i}-1-\sigma(\xi)\right)^{\frac{\alpha_{i}-1}{2}}}{\Gamma\left(\alpha_{i}\right)} F_{i}^{*}\left[u\left(t_{j}, \xi\right)\right]\right] \\
& -\phi_{q}\left[\sum_{\xi=\alpha_{i}+\beta_{i}-1}^{t_{i}+\beta_{i}-1} \frac{\left(t_{i}+\alpha_{i}+\beta_{i}-1-\sigma(\xi)\right)^{\alpha_{i}-1}}{\Gamma\left(\alpha_{i}\right)} F_{i}^{*}\left[v\left(t_{j}, \xi\right)\right]\right] \mid \\
\leq & (q-1) \mathcal{M}_{i}^{q-2} \sum_{\xi=\alpha_{i}+\beta_{i}-1}^{t_{i}+\beta_{i}-1} \frac{\left(t_{i}+\alpha_{i}+\beta_{i}-1-\sigma(\xi)\right)^{\alpha_{i}-1}}{\Gamma\left(\alpha_{i}\right)}\left|F_{i}^{*}\left[u\left(t_{j}, \xi\right)\right]-F_{i}^{*}\left[v\left(t_{j}, \xi\right)\right]\right| \\
\leq & (q-1) \mathcal{M}_{i}^{q-2} \frac{t_{i}^{\alpha_{i}}}{\Gamma\left(\alpha_{i}+1\right)}\left[L_{i}\left\|\Delta^{\gamma_{i}} u_{i}-\Delta^{\gamma_{i}} v_{i}\right\|+M_{j}\left\|\Psi^{\omega_{i}} u_{i}-\Psi^{\omega_{j}} v_{i}\right\|+N_{j}\left\|u_{j}-v_{j}\right\|\right] \\
\leq & (q-1) \mathcal{M}_{i}^{q-2} \frac{t_{i}^{\alpha_{i}}}{\Gamma\left(\alpha_{i}+1\right)}\left[L_{i}\left\|\Delta^{\gamma_{i}} u_{i}-\Delta^{\gamma_{i}} v_{i}\right\|+\left(N_{j}+M_{j} \varphi_{j}^{o} \frac{\left(T+\omega_{j}+2\right)^{\omega_{j}}}{\Gamma\left(\omega_{j}+1\right)}\right)\left\|u_{j}-v_{j}\right\|\right] \\
\leq & (q-1) \mathcal{M}_{i}^{q-2} \frac{t_{i}^{\alpha_{i}}}{\Gamma\left(\alpha_{i}+1\right)}\left[L_{i}+N_{j}+M_{j} \varphi_{j}^{o} \frac{\left(T+\omega_{j}+2\right)^{\omega_{j}}}{\Gamma\left(\omega_{j}+1\right)}\right] \\
& \times\left\|\left(u_{1}-v_{1}, u_{2}-v_{2}\right)\right\|
\end{aligned}
$$

Then, by (3.19) and (3.20), we have

$$
\begin{aligned}
& \left|\left(\mathcal{T}_{1}\left(u_{1}, u_{2}\right)\right)\left(t_{1}, t_{2}\right)-\left(\mathcal{T}_{1}\left(v_{1}, v_{2}\right)\right)\left(t_{1}, t_{2}\right)\right| \\
& \quad \leq\left\|\left(u_{1}-v_{1}, u_{2}-v_{2}\right)\right\|_{\mathcal{U}} \max \left\{\mathcal{K}_{1} \mathcal{M}_{1}^{q-2} \bar{\Omega}_{1}+\mathcal{K}_{2} \mathcal{M}_{2}^{q-2} \bar{\Theta}_{2}\right\}, \\
& \quad\left|\left(\mathcal{T}_{2}\left(u_{1}, u_{2}\right)\right)\left(t_{1}, t_{2}\right)-\left(\mathcal{T}_{2}\left(v_{1}, v_{2}\right)\right)\left(t_{1}, t_{2}\right)\right| \\
& \quad \leq\left\|\left(u_{1}-v_{1}, u_{2}-v_{2}\right)\right\|_{\mathcal{U}} \max \left\{\mathcal{K}_{1} \mathcal{M}_{1}^{q-2} \bar{\Theta}_{1}+\mathcal{K}_{2} \mathcal{M}_{2}^{q-2} \bar{\Omega}_{2}\right\} .
\end{aligned}
$$

Similarly as in Theorem 3.1, we obtain

$$
\begin{aligned}
& \left|\Delta^{\gamma_{1}}\left(\mathcal{T}_{1}\left(u_{1}, u_{2}\right)\right)\left(t_{1}, t_{2}\right)-\Delta^{\gamma_{2} 1}\left(\mathcal{T}_{1}\left(v_{1}, v_{2}\right)\right)\left(t_{1}, t_{2}\right)\right| \\
& \quad \leq\left\|\left(u_{1}-v_{1}, u_{2}-v_{2}\right)\right\|_{\mathcal{U}}\left\{\mathcal{K}_{1} \mathcal{M}_{1}^{q-2} \frac{(T+2) \frac{-\gamma_{1}}{\Gamma\left(1-\gamma_{1}\right)}}{\Omega_{1}}+\mathcal{K}_{2} \mathcal{M}_{2}^{q-2} \frac{(T+2) \frac{-\gamma_{1}}{\Gamma\left(1-\gamma_{1}\right)}}{\Theta_{2}}\right\}, \\
& \left|\Delta^{\gamma_{2}}\left(\mathcal{T}_{2}\left(u_{1}, u_{2}\right)\right)\left(t_{1}, t_{2}\right)-\Delta^{\gamma_{2}}\left(\mathcal{T}_{2}\left(v_{1}, v_{2}\right)\right)\left(t_{1}, t_{2}\right)\right| \\
& \quad \leq\left\|\left(u_{1}-v_{1}, u_{2}-v_{2}\right)\right\|_{\mathcal{U}}\left\{\mathcal{K}_{1} \mathcal{M}_{1}^{q-2} \frac{(T+2) \frac{-\gamma_{1}}{\Gamma\left(1-\gamma_{1}\right)}}{\Theta_{1}}+\mathcal{K}_{2} \mathcal{M}_{2}^{q-2} \frac{(T+2) \frac{-\gamma_{1}}{\Gamma\left(1-\gamma_{1}\right)}}{\Omega_{2}}\right\} .
\end{aligned}
$$

By (3.36) and (3.37), we have

$$
\begin{gathered}
\left\|\left(\mathcal{T}\left(u_{1}, u_{2}\right)\right)-\left(\mathcal{T}\left(v_{1}, v_{2}\right)\right)\right\|_{\mathcal{C}_{1}} \\
\quad<\left\|\left(u_{1}-v_{1}, u_{2}-v_{2}\right)\right\|_{\mathcal{U}}
\end{gathered}
$$




$$
\begin{aligned}
& \times \max \left\{\mathcal{K}_{1} \mathcal{M}_{1}^{q-2} \bar{\Theta}_{1}+\mathcal{K}_{2} \mathcal{M}_{2}^{q-2} \bar{\Omega}_{2}, \mathcal{K}_{1} \mathcal{M}_{1}^{q-2} \frac{(T+2) \frac{-\gamma_{1}}{\Gamma\left(1-\gamma_{1}\right)}}{\Omega_{1}}\right. \\
& \left.+\mathcal{K}_{2} \mathcal{M}_{2}^{q-2} \frac{(T+2) \frac{-\gamma_{1}}{\Gamma\left(1-\gamma_{1}\right)}}{\Theta_{2}}\right\}
\end{aligned}
$$

By (3.35) and (3.38), we have

$$
\begin{aligned}
& \left\|\left(\mathcal{T}\left(u_{1}, u_{2}\right)\right)-\left(\mathcal{T}\left(v_{1}, v_{2}\right)\right)\right\|_{\mathcal{C}_{2}} \\
& <\left\|\left(u_{1}-v_{1}, u_{2}-v_{2}\right)\right\|_{\mathcal{U}} \\
& \quad \times \max \left\{\mathcal{K}_{1} \mathcal{M}_{1}^{q-2} \bar{\Omega}_{1}+\mathcal{K}_{2} \mathcal{M}_{2}^{q-2} \bar{\Theta}_{2}, \mathcal{K}_{1} \mathcal{M}_{1}^{q-2} \frac{(T+2) \frac{-\gamma_{1}}{\Gamma\left(1-\gamma_{1}\right)}}{\Theta_{1}}\right. \\
& \left.\quad+\mathcal{K}_{2} \mathcal{M}_{2}^{q-2} \frac{(T+2) \frac{-\gamma_{1}}{\Gamma\left(1-\gamma_{1}\right)}}{\Omega_{2}}\right\} .
\end{aligned}
$$

Therefore, by (3.39) and (3.40), we can conclude that

$$
\begin{aligned}
& \left\|\left(\mathcal{T}\left(u_{1}, u_{2}\right)\right)-\left(\mathcal{T}\left(v_{1}, v_{2}\right)\right)\right\|_{\mathcal{U}} \\
& <\left\|\left(u_{1}-v_{1}, u_{2}-v_{2}\right)\right\|_{\mathcal{U}} \\
& \times \max \left\{\mathcal{K}_{1} \mathcal{M}_{1}^{q-2} \bar{\Theta}_{1}+\mathcal{K}_{2} \mathcal{M}_{2}^{q-2} \bar{\Omega}_{2},\right. \\
& \mathcal{K}_{1} \mathcal{M}_{1}^{q-2} \frac{(T+2) \frac{-\gamma_{1}}{\Gamma\left(1-\gamma_{1}\right)}}{\Omega_{1}}+\mathcal{K}_{2} \mathcal{M}_{2}^{q-2} \frac{(T+2) \frac{-\gamma_{1}}{\Gamma\left(1-\gamma_{1}\right)}}{\bar{\Theta}_{2}}, \\
& \left.\mathcal{K}_{1} \mathcal{M}_{1}^{q-2} \bar{\Omega}_{1}+\mathcal{K}_{2} \mathcal{M}_{2}^{q-2} \bar{\Theta}_{2}, \mathcal{K}_{1} \mathcal{M}_{1}^{q-2} \frac{(T+2) \frac{-\gamma_{2}}{\Gamma\left(1-\gamma_{2}\right)}}{\bar{\Theta}_{1}}+\mathcal{K}_{2} \mathcal{M}_{2}^{q-2} \frac{(T+2) \frac{-\gamma_{2}}{\Gamma\left(1-\gamma_{2}\right)}}{\bar{\Omega}_{2}}\right\} \\
& =\left\|\left(u_{1}-v_{1}, u_{2}-v_{2}\right)\right\|_{\mathcal{U}} \Upsilon \text {. }
\end{aligned}
$$

By (3.30), we can conclude that $\mathcal{T}$ is a contraction mapping. Hence, by the Banach fixed point theorem, $\mathcal{T}$ has a fixed point, which is a unique solution of problem (1.1)-(1.2).

\section{Some examples}

In this section, we consider some examples to illustrate our main result.

Example 4.1 Consider the following fractional sum boundary value problem:

$$
\begin{aligned}
\Delta_{C}^{\frac{1}{2}} & {\left[\phi_{\frac{5}{2}}\left(\Delta_{C}^{\frac{2}{3}} u_{1}\right)\right](t) } \\
= & F_{1}\left[t+\frac{1}{6}, t+\frac{1}{8}, \Delta^{\frac{1}{3}} u_{1}\left(t+\frac{5}{6}\right), \Psi^{\frac{1}{4}} u_{2}\left(t+\frac{3}{8}\right), u_{2}\left(t+\frac{1}{8}\right)\right], \\
\Delta_{C}^{\frac{3}{4}} & {\left[\phi_{\frac{5}{2}}\left(\Delta_{C}^{\frac{3}{8}} u_{2}\right)\right](t) } \\
& =F_{2}\left[t+\frac{1}{6}, t+\frac{1}{8}, \Delta^{\frac{2}{3}} u_{2}\left(t+\frac{11}{24}\right), \Psi^{\frac{3}{4}} u_{1}\left(t+\frac{11}{12}\right), u_{1}\left(t+\frac{1}{6}\right)\right],
\end{aligned}
$$


subject to nonlocal fractional sum boundary conditions of the form

$$
\begin{array}{ll}
\Delta_{C}^{\frac{2}{3}} u_{1}\left(-\frac{1}{2}\right)=0, & u_{1}\left(\frac{67}{6}\right)=3 \Delta^{-\frac{2}{5}} e^{2 \sin \left(\frac{221}{40} \pi\right)} u_{2}\left(\frac{221}{40}\right), \\
\Delta_{C}^{\frac{3}{4}} u_{1}\left(-\frac{1}{4}\right)=0, & u_{1}\left(\frac{89}{8}\right)=2 \Delta^{-\frac{1}{4}} e^{\cos \left(\frac{41}{12} \pi\right)} u_{1}\left(\frac{41}{12}\right),
\end{array}
$$

where $t \in \mathbb{N}_{0,10}$. Functions $F_{1}, F_{2}$ are determined by

$$
\begin{gathered}
F_{1}\left[t_{1}, t_{2}, \Delta^{\frac{1}{3}} u_{1}\left(t_{1}-\frac{2}{3}\right), \Psi^{\frac{1}{4}} u_{2}\left(t_{2}+\frac{1}{4}\right), u_{2}\left(t_{2}\right)\right] \\
=3 t_{1}^{\frac{2}{1}}\left[1+\frac{1}{50,000 e^{7}} \sin ^{2}\left(\Delta^{\frac{1}{3}} u_{1}\left(t_{1}-\frac{2}{3}\right)\right)\right] \\
+2 t_{2}^{2}\left[1+\frac{1}{40,000 e^{8}} \cos ^{2}\left(\Psi^{\frac{1}{4}} u_{2}\left(t_{2}+\frac{1}{4}\right)\right)\right] \\
+t_{2}^{\frac{2}{2}}\left[1+\frac{1}{60,000 e^{6}} \sin ^{2}\left(u_{2}\left(t_{2}\right)\right)\right], \\
F_{2}\left[t_{1}, t_{2}, \Delta^{\frac{2}{3}} u_{2}\left(t_{2}-\frac{1}{3}\right), \Psi^{\frac{3}{4}} u_{1}\left(t_{1}+\frac{3}{4}\right), u_{1}\left(t_{1}\right)\right] \\
=2 t_{2}^{\frac{2}{2}}\left[1+\frac{1}{60,000 e^{6}} \sin ^{2}\left(\Delta^{\frac{2}{3}} u_{2}\left(t_{2}-\frac{1}{3}\right)\right)\right] \\
+2 t_{1}^{2}\left[1+\frac{1}{50,000 e^{7}} \cos ^{2}\left(\Psi^{\frac{3}{4}} u_{1}\left(t_{1}+\frac{3}{4}\right)\right)\right] \\
+3 t_{1}^{2}\left[1+\frac{1}{40,000 e^{8}} \sin ^{2}\left(u_{1}\left(t_{1}\right)\right)\right],
\end{gathered}
$$

and

$$
\begin{aligned}
& \Psi^{\frac{3}{4}} u_{1}\left(t_{1}+\frac{3}{4}\right)=\frac{1}{\Gamma\left(\frac{3}{4}\right)} \sum_{s=-\frac{19}{12}}^{t_{1}-\frac{3}{4}}\left(t_{1}-\sigma(s)\right)^{\frac{3}{4}-1} \frac{e^{-s}}{\left(t_{1}+10\right)^{3}} u_{1}\left(t_{1}+\frac{3}{4}\right), \\
& \Psi^{\frac{1}{4}} u_{2}\left(t_{2}+\frac{1}{4}\right)=\frac{1}{\Gamma\left(\frac{1}{4}\right)} \sum_{s=-\frac{9}{8}}^{t_{1}-\frac{1}{4}}\left(t_{2}-\sigma(s)\right)^{\frac{1}{4}-1} \frac{e^{-s}}{\left(t_{2}+20\right)^{2}} u_{2}\left(t_{2}+\frac{1}{4}\right) .
\end{aligned}
$$

Here, $p=\frac{5}{2}, q=\frac{5}{3}, \alpha_{1}=\frac{1}{2}, \alpha_{2}=\frac{3}{4}, \beta_{1}=\frac{2}{3}, \beta_{2}=\frac{3}{8}, \gamma_{1}=\frac{1}{3}, \gamma_{2}=\frac{2}{3}, \omega_{1}=\frac{3}{4}, \omega_{2}=\frac{1}{4}, \theta_{1}=\frac{1}{4}$, $\theta_{2}=\frac{2}{5}, \eta_{1}=\frac{19}{6}, \eta_{2}=\frac{41}{8}, \lambda_{1}=2, \lambda_{2}=3, T=10, g_{1}\left(t_{1}\right)=e^{\cos t_{1} \pi}, g_{2}\left(t_{2}\right)=e^{2 \sin t_{2} \pi}, \varphi_{1}\left(t_{1}, s\right)=$ $\frac{e^{-s}}{\left(t_{1}+10\right)^{3}}, \varphi_{2}=\frac{e^{-s}}{\left(t_{2}+20\right)^{2}}$, and $\varphi_{1}^{o}=\frac{216}{166,375 e^{1 / 6}} \approx 0.0011, \varphi_{2}^{o}=\frac{64}{23,409 e^{1 / 8}} \approx 0.0024$.

Let $t_{1} \in \mathbb{N}_{-\frac{5}{6}, \frac{67}{6}}$ and $t_{2} \in \mathbb{N}_{-\frac{7}{8}, \frac{89}{8}}$. Taking $\chi_{1}=3, \chi_{2}=2$ and $1=\delta<\frac{1}{2-q}=3$, we have

$$
\begin{aligned}
& \chi_{1} \Delta_{C}^{\frac{1}{2}}\left(t_{1}^{1 / 2}\right) \leq 3 t_{1}^{2} \leq 3 t_{1}^{\frac{2}{2}}+3 t_{2}^{\frac{2}{2}} \leq F_{1}\left[t_{1}, t_{2}, x, y, z\right], \\
& \chi_{2} \Delta_{C}^{\frac{3}{4}}\left(t_{2}^{3 / 4}\right) \leq 2 t_{2}^{2} \leq 2 t_{2}^{\frac{2}{2}}+5 t_{1}^{\frac{2}{1}} \leq F_{2}\left[t_{1}, t_{2}, x, y, z\right] .
\end{aligned}
$$

Thus, (H1) holds. 
For $\left(u_{1}, u_{2}\right),\left(v_{1}, v_{2}\right) \in \mathcal{C}$, we have

$$
\begin{aligned}
& \left|F_{1}\left[t_{1}, t_{2}, \Delta^{\frac{1}{3}} u_{1}, \Psi^{\frac{1}{4}} u_{2}, u_{2}\right]-F_{1}\left[t_{1}, t_{2}, \Delta^{\frac{1}{3}} v_{1}, \Psi^{\frac{1}{4}} v_{2}, v_{2}\right]\right| \\
& \quad \leq \frac{3 t_{1}^{\frac{2}{2}}}{50,000 e^{7}}\left|\Delta^{\frac{1}{3}} u_{1}-\Delta^{\frac{1}{3}} v_{1}\right|+\frac{2 t_{2}^{\frac{2}{2}}}{40,000 e^{8}}\left|\Psi^{\frac{1}{4}} u_{2}-\Psi^{\frac{1}{4}} v_{2}\right|+\frac{t_{2}^{\frac{2}{2}}}{60,000 e^{6}}\left|u_{2}-v_{2}\right|, \\
& \left|F_{2}\left[t_{1}, t_{2}, \Delta^{\frac{2}{3}} u_{2}, \Psi^{\frac{3}{4}} u_{1}, u_{1}\right]-F_{2}\left[t_{1}, t_{2}, \Delta^{\frac{2}{3}} v_{2}, \Psi^{\frac{3}{4}} v_{1}, v_{1}\right]\right| \\
& \quad \leq \frac{2 t_{2}^{\frac{2}{2}}}{60,000 e^{6}}\left|\Delta^{\frac{2}{3}} u_{2}-\Delta^{\frac{2}{3}} v_{2}\right|+\frac{2 t_{1}^{\frac{2}{1}}}{50,000 e^{7}}\left|\Psi^{\frac{3}{4}} u_{1}-\Psi^{\frac{3}{4}} v_{1}\right|+\frac{3 t_{2}^{\frac{2}{2}}}{40,000 e^{8}}\left|u_{1}-v_{1}\right| .
\end{aligned}
$$

Thus, (H2) holds with $L_{1}=6.211 \times 10^{-6}, L_{2}=9.307 \times 10^{-6}, M_{1}=4.141 \times 10^{-6}, M_{2}=$ $1.889 \times 10^{-6}, N_{1}=2.856 \times 10^{-6}$, and $N_{2}=4.653 \times 10^{-6}$.

Since $\frac{1}{e} \leq g_{1}\left(t_{1}\right) \leq e$ and $\frac{1}{e^{2}} \leq g_{2}\left(t_{2}\right) \leq e^{2}$.

Thus, (H3) holds with $g_{1}=\frac{1}{e}, g_{2}=\frac{1}{e^{2}}$ and $G_{1}=e, G_{2}=e^{2}$.

Finally, we find that

$$
\begin{aligned}
& \Lambda \geq 0.029, \quad \mathcal{K}_{1}=0.286, \quad \mathcal{K}_{2}=0.574, \quad \Omega_{1}=3783.803 \\
& \Omega_{2}=31,848.989, \quad \Theta_{1}=39,305.323, \quad \text { and } \quad \Theta_{2}=55,288.515
\end{aligned}
$$

Therefore, we have

$$
\Phi=\max \{0.446,0.410,0.130,0.030\}=0.446<1
$$

Hence, by Theorem 3.1, boundary value problem (4.1)-(4.2) has a unique solution.

Example 4.2 Consider the following fractional sum boundary value problem:

$$
\begin{aligned}
& \Delta_{C}^{\frac{1}{2}}\left[\phi_{\frac{5}{2}}\left(\Delta_{C}^{\frac{2}{3}} u_{1}\right)\right](t) \\
& \quad=H_{1}\left[t+\frac{1}{6}, t+\frac{1}{8}, \Delta^{\frac{1}{3}} u_{1}\left(t+\frac{5}{6}\right), \Psi^{\frac{1}{4}} u_{2}\left(t+\frac{3}{8}\right), u_{2}\left(t+\frac{1}{8}\right)\right], \\
& \Delta_{C}^{\frac{3}{4}}\left[\phi_{\frac{5}{2}}\left(\Delta_{C}^{\frac{3}{8}} u_{2}\right)\right](t) \\
& \quad=H_{2}\left[t+\frac{1}{6}, t+\frac{1}{8}, \Delta^{\frac{2}{3}} u_{2}\left(t+\frac{11}{24}\right), \Psi^{\frac{3}{4}} u_{1}\left(t+\frac{11}{12}\right), u_{1}\left(t+\frac{1}{6}\right)\right],
\end{aligned}
$$

where $t \in \mathbb{N}_{0,10}$, and the nonlocal fractional sum boundary conditions satisfy (4.2). Functions $H_{1}, H_{2}$ are determined by

$$
\begin{aligned}
& H_{1}\left[t_{1}, t_{2}, \Delta^{\frac{1}{3}} u_{1}\left(t_{1}-\frac{2}{3}\right), \Psi^{\frac{1}{4}} u_{2}\left(t_{2}+\frac{1}{4}\right), u_{2}\left(t_{2}\right)\right] \\
& =\frac{3 t_{1}^{\frac{2}{2}}}{500,000 e^{7}} \sin ^{2}\left(\Delta^{\frac{1}{3}} u_{1}\left(t_{1}-\frac{2}{3}\right)\right) \\
& \quad+\frac{2 t_{2}^{\frac{2}{2}}}{400,000 e^{8}}\left[\cos ^{2}\left(\Psi^{\frac{1}{4}} u_{2}\left(t_{2}+\frac{1}{4}\right)\right)+\sin ^{2}\left(u_{2}\left(t_{2}\right)\right)\right], \\
& H_{2}\left[t_{1}, t_{2}, \Delta^{\frac{2}{3}} u_{2}\left(t_{2}-\frac{1}{3}\right), \Psi^{\frac{3}{4}} u_{1}\left(t_{1}+\frac{3}{4}\right), u_{1}\left(t_{1}\right)\right]
\end{aligned}
$$




$$
\begin{aligned}
= & \frac{2 t_{2}^{\frac{2}{2}}}{6,000,000 e^{6}} \sin ^{2}\left(\Delta^{\frac{2}{3}} u_{2}\left(t_{2}-\frac{1}{3}\right)\right) \\
& +\frac{t_{1}^{2}}{5,000,000 e^{7}}\left[\cos ^{2}\left(\Psi^{\frac{3}{4}} u_{1}\left(t_{1}+\frac{3}{4}\right)\right)+\sin ^{2}\left(u_{1}\left(t_{1}\right)\right)\right]
\end{aligned}
$$

where $\Psi^{\frac{3}{4}} \mathcal{u}_{1}, \Psi^{\frac{1}{4}} \mathcal{u}_{2}$ are defined as (4.3) and (4.4), respectively.

Let $t_{1} \in \mathbb{N}_{-\frac{5}{6}, \frac{67}{6}}$ and $t_{2} \in \mathbb{N}_{-\frac{7}{8}, \frac{89}{8}}$. Using $g_{1}\left(t_{1}, t_{2}\right)=\frac{3 t_{1}^{2}}{500,000 e^{7}}+\frac{2 t_{2}^{2}}{400,000 e^{8}}$ and $g_{2}\left(t_{1}, t_{2}\right)=$ $\frac{2 t_{2}^{2}}{6,000,000 e^{6}}+\frac{t_{1}^{2}}{5,000,000 e^{7}}$, we have

$$
\mathcal{M}_{1}=0.000709 \text { and } \mathcal{M}_{2}=0.00272 .
$$

For $\left(u_{1}, u_{2}\right),\left(v_{1}, v_{2}\right) \in \mathcal{C}$, we have

$$
\begin{aligned}
& \left|F_{1}\left[t_{1}, t_{2}, \Delta^{\frac{1}{3}} u_{1}, \Psi^{\frac{1}{4}} u_{2}, u_{2}\right]-F_{1}\left[t_{1}, t_{2}, \Delta^{\frac{1}{3}} v_{1}, \Psi^{\frac{1}{4}} v_{2}, v_{2}\right]\right| \\
& \quad \leq \frac{3 t_{1}^{\frac{2}{2}}}{500,000 e^{7}}\left|\Delta^{\frac{1}{3}} u_{1}-\Delta^{\frac{1}{3}} v_{1}\right|+\frac{2 t_{2}^{\frac{2}{2}}}{40000 e^{8}}\left[\left|\Psi^{\frac{1}{4}} u_{2}-\Psi^{\frac{1}{4}} v_{2}\right|+\left|u_{2}-v_{2}\right|\right], \\
& \left|F_{2}\left[t_{1}, t_{2}, \Delta^{\frac{2}{3}} u_{2}, \Psi^{\frac{3}{4}} u_{1}, u_{1}\right]-F_{2}\left[t_{1}, t_{2}, \Delta^{\frac{2}{3}} v_{2}, \Psi^{\frac{3}{4}} v_{1}, v_{1}\right]\right| \\
& \quad \leq \frac{2 t_{2}^{\frac{2}{2}}}{600,000 e^{6}}\left|\Delta^{\frac{2}{3}} u_{2}-\Delta^{\frac{2}{3}} v_{2}\right|+\frac{2 t_{1}^{\frac{2}{2}}}{500,000 e^{7}}\left[\left|\Psi^{\frac{3}{4}} u_{1}-\Psi^{\frac{3}{4}} v_{1}\right|+\left|u_{1}-v_{1}\right|\right] .
\end{aligned}
$$

Thus, (H2) holds with $L_{1}=6.211 \times 10^{-7}, L_{2}=9.307 \times 10^{-7}, M_{1}=N_{1}=2.070 \times 10^{-7}$, and $M_{2}=N_{2}=9.447 \times 10^{-8}$.

From Example 4.1, we get $\Lambda \geq 0.029, g_{1}=\frac{1}{e}, g_{2}=\frac{1}{e^{2}}$ and $G_{1}=e, G_{2}=e^{2}$.

Finally, we find that

$$
\begin{aligned}
& \mathcal{K}_{1}=5.385 \times 10^{-7}, \quad \mathcal{K}_{2}=8.261 \times 10^{-7}, \quad \bar{\Omega}_{1}=4993.134, \\
& \bar{\Omega}_{2}=33,202.614, \quad \bar{\Theta}_{1}=44,000.064 \text { and } \bar{\Theta}_{2}=77,432.180 \text {. }
\end{aligned}
$$

Hence,

$$
\Upsilon=\max \{0.285,0.019,0.489,0.155\}=0.489<1 .
$$

From Theorem 3.3, we can conclude that boundary value problem (4.5) and (4.2) has a unique solution.

\section{Conclusions}

We have proved existence and uniqueness results of the nonlocal fractional sum boundary value problem for a coupled system of fractional sum-difference equations with $p$ Laplacian operator (1.1)-(1.2) by using the Banach fixed point theorem. Our problem contains both Riemann-Liouville and Caputo fractional difference with five fractional differences and four fractional sums. 


\section{Funding}

This research was funded by King Mongkut's University of Technology North Bangkok. Contract no. KMUTNB-61-KNOW-028.

\section{Availability of data and materials}

Not applicable.

\section{Ethics approval and consent to participate}

Not applicable.

\section{Competing interests}

The authors declare that they have no competing interests.

\section{Consent for publication}

Not applicable.

\section{Authors' contributions}

All authors read and approved the final manuscript.

\section{Author details}

1'Department of Mathematics, Faculty of Science, Kasetsart University, Bangkok 10900, Thailand. ${ }^{2}$ Department of Mathematics, Faculty of Applied Science, King Mongkut's University of Technology North Bangkok, Bangkok 10800, Thailand. ${ }^{3}$ Mathematics Department, Faculty of Science and Technology, Suan Dusit University, Bangkok 10300, Thailand.

\section{Publisher's Note}

Springer Nature remains neutral with regard to jurisdictional claims in published maps and institutional affiliations.

Received: 6 May 2020 Accepted: 8 July 2020 Published online: 16 July 2020

\section{References}

1. Goodrich, C.S., Peterson, A.C.: Discrete Fractional Calculus. Springer, New York (2015)

2. Wu, G.C., Baleanu, D.: Discrete fractional logistic map and its chaos. Nonlinear Dyn. 75, 283-287 (2014)

3. Wu, G.C., Baleanu, D.: Chaos synchronization of the discrete fractional logistic map. Signal Process. 102, 96-99 (2014)

4. Wu, G.C., Baleanu, D., Xie, H.P., Chen, F.L.: Chaos synchronization of fractional chaotic maps based on stability results. Physica A 460, 374-383 (2016)

5. Atici, F.M., Eloe, P.W.: Initial value problems in discrete fractional calculus. Proc. Am. Math. Soc. 137(3), 981-989 (2009)

6. Atici, F.M., Eloe, P.W.: Two-point boundary value problems for finite fractional difference equations. J. Differ. Equ. Appl. $17,445-456(2011)$

7. Abdeljawad, T: On Riemann and Caputo fractional differences. Comput. Math. Appl. 62(3), 1602-1611 (2011)

8. Jia, B., Erbe, L., Peterson, A.: Two monotonicity results for nabla and delta fractional differences. Arch. Math. 104, 589-597 (2015)

9. Jia, B., Erbe, L., Peterson, A.: Convexity for nabla and delta fractional differences. J. Differ. Equ. Appl. 21, 360-373 (2015)

10. Ferreira, R.A.C.: Existence and uniqueness of solution to some discrete fractional boundary value problems of order less than one. J. Differ. Equ. Appl. 19, 712-718 (2013)

11. Ferreira, R.A.C., Goodrich, C.S.: Positive solution for a discrete fractional periodic boundary value problem. Dyn. Contin. Discrete Impuls. Syst., Ser. A Math. Anal. 19, 545-557 (2012)

12. Goodrich, C.S.: Existence and uniqueness of solutions to a fractional difference equation with nonlocal conditions. Comput. Math. Appl. 61, 191-202 (2011)

13. Goodrich, C.S.: On a discrete fractional three-point boundary value problem. J. Differ. Equ. Appl. 18, 397-415 (2012)

14. Erbe, L., Goodrich, C.S., Jia, B., Peterson, A.: Survey of the qualitative properties of fractional difference operators: monotonicity, convexity, and asymptotic behavior of solutions. Adv. Differ. Equ. 2016, 43 (2016)

15. Chen, Y., Tang, X.: Three difference between a class of discrete fractional and integer order boundary value problems. Commun. Nonlinear Sci. 19(12), 4057-4067 (2014)

16. LV, W., Feng, J.: Nonlinear discrete fractional mixed type sum-difference equation boundary value problems in Banach spaces. Adv. Differ. Equ. 2014, 184 (2014)

17. Lv, W: Existence of solutions for discrete fractional boundary value problems with a $p$-Laplacian operator. Adv. Differ. Equ. 2012, 163 (2012)

18. Kang, S.G., Li, Y., Chen, H.Q.: Positive solutions to boundary value problems of fractional difference equations with nonlocal conditions. Adv. Differ. Equ. 2014, 7 (2014)

19. Dong, W., Xu, J., Regan, D.O.: Solutions for a fractional difference boundary value problem. Adv. Differ. Equ. 2013, 319 (2013)

20. Holm, M.: Sum and difference compositions in discrete fractional calculus. CUBO 13(3), 153-184 (2011)

21. Sitthiwirattham, T., Tariboon, J., Ntouyas, S.K.: Existence results for fractional difference equations with three-point fractional sum boundary conditions. Discrete Dyn. Nat. Soc. 2013, Article ID 104276 (2013)

22. Sitthiwirattham, T., Tariboon, J., Ntouyas, S.K.: Boundary value problems for fractional difference equations with three-point fractional sum boundary conditions. Adv. Differ. Equ. 2013, 296 (2013)

23. Sitthiwirattham, T.: Existence and uniqueness of solutions of sequential nonlinear fractional difference equations with three-point fractional sum boundary conditions. Math. Methods Appl. Sci. 38, 2809-2815 (2015)

24. Chasreechai, S., Kiataramkul, C., Sitthiwirattham, T:: On nonlinear fractional sum-difference equations via fractional sum boundary conditions involving different orders. Math. Probl. Eng. 2015, Article ID 519072 (2015) 
25. Reunsumrit, J., Sitthiwirattham, T.: Positive solutions of three-point fractional sum boundary value problem for Caputo fractional difference equations via an argument with a shift. Positivity 20(4), 861-876 (2016)

26. Reunsumrit, J., Sitthiwirattham, T.: On positive solutions to fractional sum boundary value problems for nonlinear fractional difference equations. Math. Methods Appl. Sci. 39(10), 2737-2751 (2016)

27. Soontharanon, J., Jasthitikulchai, N., Sitthiwirattham, T.: Nonlocal fractional sum boundary value problems for mixed types of Riemann-Liouville and Caputo fractional difference equations. Dyn. Syst. Appl. 25, 409-414 (2016)

28. Laoprasittichok, S., Sitthiwirattham, T.: On a fractional difference-sum boundary value problems for fractional difference equations involving sequential fractional differences via different orders. J. Comput. Anal. Appl. 23(6), 1097-1111 (2017)

29. Kaewwisetkul, B., Sitthiwirattham, T.: On nonlocal fractional sum-difference boundary value problems for Caputo fractional functional difference equations with delay. Adv. Differ. Equ. 2017, 219 (2017)

30. Reunsumrit, J., Sitthiwirattham, T.: A new class of four-point fractional sum boundary value problems for nonlinear sequential fractional difference equations involving shift operators. Kragujev. J. Math. 42(3), 371-387 (2018)

31. Chasreechai, S., Sitthiwirattham, T.: Existence results of initial value problems for hybrid fractional sum-difference equations. Discrete Dyn. Nat. Soc. 2018, Article ID 5268528 (2018)

32. Chasreechai, S., Sitthiwirattham, T.: On separate fractional sum-difference boundary value problems with $n$-point fractional sum-difference boundary conditions via arbitrary different fractional orders. Mathematics 2019(7), Article ID 471 (2019)

33. Khan, H., Chen, W., Khan, A., Khan, T.S., Al-Madlal, Q.M.: Hyers-Ulam stability and existence criteria for coupled fractional differential equations involving p-Laplacian operator. Adv. Differ. Equ. 2018, 455 (2018)

34. Khan, H., Tunc, C., Chen, W., Khan, A.: Existence theorems and Hyers-Ulam stability for a class of hybrid fractional differential equations with $p$-Laplacian operator. J. Appl. Anal. Comput. 8(4), 1211-1226 (2018)

35. Khan, H., Chen, W., Sun, H.: Analysis of positive solution and Hyers-Ulam stability for a class of singular fractional differential equations with p-Laplacian in Banach space. Math. Methods Appl. Sci. 41(9), 3430-3440 (2018)

36. Khan, H., Li, Y., Chen, W., Baleanu, D., Khan, A.: Existence theorems and Hyers-Ulam stability for a coupled system of fractional differential equations with $p$-Laplacian operator. Bound. Value Probl. 2018, 157 (2017)

37. Jafari, H., Baleanu, D., Khan, H., Khan, R.A., Khan, A.: Existence criterion for the solutions of fractional order $p$-Laplacian boundary value problems. Bound. Value Probl. 2015, 164 (2015)

38. Khan, A., Khan, H., Gómez-Aguilar, J.F., Abdeljawad, T.: Existence and Hyers-Ulam stability for a nonlinear singular fractional differential equations with Mittag-Leffler kernel. Chaos Solitons Fractals 127, 422-427 (2019)

39. Khan, H., Jarad, F., Abdeljawad, T., Khan, A.: A singular ABC-fractional differential equation with $p$-Laplacian operator. Chaos Solitons Fractals 129, 56-61 (2019)

40. Hu, L., Shuqin, Z.: Existence results for a coupled system of fractional differential equations with $p$-Laplacian operator and infinite-point boundary conditions. Bound. Value Probl. 2017, 88 (2017)

41. Hao, X., Wang, H., Liu, L., Cui, Y.: Positive solutions for a system of nonlinear fractional nonlocal boundary value problems with parameters and p-Laplacian operator. Bound. Value Probl. 2017, 182 (2017)

42. Rao, S.N.: Multiple positive solutions for a coupled system of $p$-Laplacian fractional order three-point boundary value problems. Rocky Mt. J. Math. 49(2), 609-626 (2019)

43. Liu, Y., Xie, D., Bai, C., Yang, D.: Multiple positive solutions for a coupled system of fractional multi-point BVP with p-Laplacian operator. Adv. Differ. Equ. 2017, 168 (2017)

44. Rao, S.N., Meshari, A.: Existence of positive solutions for systems of nonlinear fractional differential equation with p-Laplacian. Asian-Eur. J. Math. 2019, 2050089 (2019)

45. Sitthiwirattham, T.: Boundary value problem for $p$-Laplacian Caputo fractional difference equations with fractional sum boundary conditions. Math. Methods Appl. Sci. 39(6), 1522-1534 (2016)

46. LV, W.: Solvability for discrete fractional boundary value problems with a $p$-Laplacian operator. Discrete Dyn. Nat. Soc. 2013, Article ID 679290 (2013)

47. Lv, W.: Existence of solutions for discrete fractional boundary value problems with a $p$-Laplacian operator. Adv. Differ. Equ. 2012, $163(2012)$

48. Pan, Y., Han, Z., Sun, S., Zhao, Y.: The existence of solutions to a system of discrete fractional boundary value problems. Abstr. Appl. Anal. 2012, Article ID 707631 (2012)

49. Goodrich, C.S.: Existence of a positive solution to a system of discrete fractional boundary value problems. Appl. Math. Comput. 217(9), 4740-4753 (2011)

50. Dahal, R., Duncan, D., Goodrich, C.S.: Systems of semipositone discrete fractional boundary value problems. J. Differ. Equ. Appl. 20(3), 473-491 (2014)

51. Goodrich, C.S.: Systems of discrete fractional boundary value problems with nonlinearities satisfying no growth conditions. J. Differ. Equ. Appl. 21(5), 437-453 (2015)

52. Goodrich, C.S.: Coupled systems of boundary value problems with nonlocal boundary conditions. Appl. Math. Lett. 41, 17-22 (2015)

53. Kunnawuttipreechachan, E., Promsakon, C., Sitthiwirattham, T.: Nonlocal fractional sum boundary value problems for a coupled system of fractional sum-difference equations. Dyn. Syst. Appl. 28(1), 73-92 (2019)

54. Promsakon, C., Chasreechai, S., Sitthiwirattham, T.: Positive solution to a coupled system of singular fractional difference equations with fractional sum boundary value conditions. Adv. Differ. Equ. 2019, Article ID 218 (2017)

55. Soontharanon, J., Chasreechai, S., Sitthiwirattham, T.: On a coupled system of fractional difference equations with nonlocal fractional sum boundary value conditions on the discrete half-line. Mathematics 2019(7), Article ID 256 (2019) 